\title{
Las elecciones democráticas de ámbito nacional durante veinte años de vida postcomunista en Bulgaria. Actitudes y percepciones acerca del cambio
}

\author{
Manuel Jacinto Roblizo Colmenero \\ Universidad de Castilla-La Mancha \\ manuel.roblizo@uclm.es
}

Recibido: 15-04-2011

Aceptado: 01-03-2012

\section{Resumen}

Una vez que han transcurrido veinte años desde la caída del muro de Berlín, una cuestión que surge es en qué medida la vida en las sociedades postcomunistas ha cambiado de manera significativa, y en qué sentido. La amplia diversidad de sociedades existente en el área posibilita una pluralidad de respuestas. Este artículo se centra en la realidad de Bulgaria, que resulta ser especialmente sugerente como consecuencia de su devenir nítidamente diferenciado del que encontramos en los Balcanes y en el conjunto del Este de Europa. La línea argumental básica es que la persistencia de las dificultades genera, inevitablemente, un sentimiento de desesperanza que aflora en el comportamiento electoral. Los resultados electorales son utilizados en el texto como una valiosa forma de observar las percepciones que los ciudadanos búlgaros se han formado a lo largo del actual periodo democrático. Hemos tratado de comprobar la validez de tres hipótesis: las motivaciones de los electores búlgaros se han sustentado en base a un análisis sobre el coste y el beneficio, a la manera propuesta por la teoría de la elección racional; las diferencias sociales aparecen como latentes en el comportamiento electoral de los ciudadanos búlgaros; las novedosas y atípicas opciones surgidas, de manera exitosa, en el panorama político búlgaro muestran un cierto grado de continuidad en sus bases sociodemográficas. A tal fin, hemos utilizado tres tipos de análisis: contextual, de correlación y de regresión lineal, con la siempre inagotable crisis económica como transfondo.

Palabras clave: transición política; comportamiento electoral; cambio político; cambio social.

Abstract. National-level democratic elections throughout 20 years of post-communist life in Bulgaria: Attitudes and perceptions about change

Twenty years after the fall of the Berlin Wall, the question arises as to what extent life in post-communist countries has significantly changed, and in what sense. The wide diversity of societies in the region offers numerous responses. This article focuses on the reality of Bulgaria today, which is of particular interest due to its particular path of development within the frame of the Balkans and Eastern Europe as a whole. We argue that the persis- 
tent difficulties in Bulgaria have led to feelings of despair which are ultimately reflected in the electoral behaviour of the country's citizens. Electoral outcomes are used to explore the perceptions of Bulgarian citizens throughout the current democratic period. In this paper we test the validity of three hypotheses, namely that the motivations of Bulgarian voters are based on a cost-benefit analysis as proposed in rational choice theory; social differences underlie Bulgarian citizens' electoral behaviour; and the novel and atypical electoral options that have successfully emerged in the Bulgarian political arena show a certain degree of continuity with regard to their corresponding sociodemographic bases. To achieve our goals, we rely on three types of analyses: contextual, correlation and linear regression, with the enduring economic crisis as a backdrop.

Keywords: political transition; electoral behaviour; political change; social change.

\section{Sumario}

1. Hipótesis y perspectivas metodológicas

2. El contexto socioeconómico: vivir en tiempos de cambio

3. El devenir de los procesos electorales: el voto en un entorno de crisis política y económica
4. Análisis estadísticos basados en datos de nivel de distrito

5. Conclusión: contrastando hipótesis a lo largo de veinte años de vida democrática

Referencias bibliográficas

\section{Hipótesis y perspectivas metodológicas}

El 10 de noviembre de 1989 — un día después de la caída del muro de Berlín - tuvo lugar una histórica sesión del Comité Central del Partido Comunista Búlgaro, que marcó el comienzo de la transición en Bulgaria. Sólo siete meses después, tenían lugar las primeras elecciones democráticas. El objetivo principal de este artículo es analizar las dos décadas iniciales de vida democrática en Bulgaria, y hacerlo sobre la base del comportamiento electoral de sus ciudadanos. De esta manera, esperamos poder ofrecer una fuente útil para contrastar, en un contexto de inacabable descontento social, las más clásicas - y, probablemente, todavía válidas - aproximaciones teóricas que han tratado de entender y de explicar las claves de la actitud de los electores ante las urnas.

En términos generales, podemos proponer tres modelos que recojan la amplia variedad de aportaciones explicativas acerca del comportamiento electoral: el de elección racional, el enfoque sociológico y el de identificación de partido (Harrop y Miller, 1987: 130-138). Ciertamente, el contexto social búlgaro de este periodo es significativamente distinto de aquél que inspirara los escritos de Anthony Downs, pero - probablemente porque la naturaleza humana es esencialmente la misma - todavía podemos considerar que «la decisión de voto se basa en una comparación del beneficio que [el votante] obtiene de las acciones del partido que gobierna durante un periodo determinado, y de los beneficios que estima que podría haber recibido si cada uno de los partidos de la oposición hubiera ocupado el poder» (Downs, 1957: 138). Desde nuestro 
punto de vista, el comportamiento electoral de los ciudadanos búlgaros resulta ser paradigmático en cuanto a los efectos políticos derivados de la persistente crisis económica y social, con su traducción en un voto de motivación fuertemente pragmática — en una línea esencialmente coincidente con el modelo de elección racional.

El llamado enfoque sociológico se encuentra representado por los conocidos textos de Seymour M. Lipset, para el que «en las democracias modernas, el conflicto entre los distintos grupos se expresa a través de partidos políticos que básicamente representan una expresión democrática de la lucha de clases» (Lipset, 1981: 230). Este punto de vista, expresado mucho antes de los cambios acontecidos en las sociedades de la Europa del Este, quedará contrastado a través de la experiencia que emana de las elecciones democráticas que han tenido lugar en Bulgaria en el periodo acotado. Más concretamente, será de particular interés la actitud hacia el cambio de cada uno de los distintos grupos sociales.

Y, finalmente, el modelo de identificación de partido, que se ocupa principalmente de la transmisión intergeneracional de valores y preferencias partidistas, no será analizado en este artículo en lo que a sus contenidos más definitorios se refiere. Sin embargo, el modelo también reflexiona acerca de hasta qué punto los votantes mantienen una identificación de partido estable. Cuando encontramos una alta volatilidad debida a circunstancias derivadas de un contexto específico — como, por ejemplo, una crisis económica一, es posible encontrar también, con una íntima vinculación a la adscripción social, un cierto grado de continuidad en el apoyo electoral hacia algunas opciones. Veremos como el panorama electoral búlgaro resulta ser paradigmático en este sentido, incluso en lo relativo a las opciones políticas más atípicas.

A la luz de estas líneas analíticas, podemos aportar algunas hipótesis que se derivan del caso búlgaro - y que trataremos de analizar en estas páginas. En primer lugar, el casi continuo movimiento de vaivén en los resultados electorales nos mostrará una naturaleza fácilmente entendible en un contexto de persistente desesperanza. En este marco, los esquemas conceptuales propios del modelo de elección racional resultarán ser de utilidad, y nuestra hipótesis radicará en que, a lo largo de estos veinte años, se produce un auge de las motivaciones basadas en componentes pragmáticos, en detrimento de aquéllos otros de base más ideológica. En segundo lugar, entendemos que el comportamiento electoral de los ciudadanos búlgaros puede ser analizado en términos grupales, en la medida en que pueden observarse en el apoyo electoral a las distintas opciones diversos cleavages socialmente relevantes. En tercer lugar, prestaremos nuestra atención a la continuidad en el apoyo electoral que pueda derivarse de la identificación hacia un partido u orientación ideológica. Ello resultará fácil cuando se trate de los partidos o las coaliciones más clásicos, pero no lo será tanto cuando se trate de aquéllos liderados por Simeón SajoniaCoburgo o por el actual primer ministro Boyko Borissov. Nuestra hipótesis será que estas novedosas opciones muestran un alto grado de continuidad entre sí, tanto en sus significaciones políticas como en el electorado que representan ante las urnas. 
De cara a verificar la primera de las hipótesis mencionadas, desarrollaremos un análisis contextual de los resultados registrados en elecciones de nivel nacional -legislativas y presidenciales - (parte 3). Obviamente, descender a datos electorales de nivel local — como sería el caso de elecciones municipales - sería algo que excedería, con mucho, las pretensiones de este artículo. Desarrollaremos nuestros análisis con elecciones que han tenido lugar durante todo el periodo democrático actual. En este sentido, las elecciones al Parlamento europeo quedarán al margen de nuestro centro de interés, dado su limitado marco comparativo.

A lo largo de estos análisis contextuales e históricos, observaremos como es asumido por científicos sociales y centros de análisis sociológicos que los cleavages sociales están en la base de los resultados electorales. Por nuestra parte, comprobaremos la segunda hipótesis indicada a través de análisis en los que algunas características sociológicas significativas de los distritos búlgaros serán correlacionadas con los resultados registrados en ellos en las sucesivas elecciones legislativas (parte 4.1). Esta forma de elección nos será de mayor utilidad para nuestros propósitos, puesto que, en el voto en elecciones presidenciales, el ingrediente más personal de candidatos y votos atenúa los rasgos más característicos de los cleavages de partidos.

Por lo que respecta a la tercera hipótesis, llevaremos a cabo un análisis de regresión (parte 4.2) en el que la hipótesis nula será que el voto 2001 MNSII (a la opción liderada por el antiguo rey Simeón II) no tiene ningún efecto sobre el voto 2009 GERB (a la opción del actual primer ministro Boyko Borissov).

Nuestra tesis central, que inspira todo el texto, será que el contexto social de inagotable desesperanza se revelará como el principal factor explicativo del comportamiento electoral observado en estos veinte años de vida democrática. En qué medida la desesperanza afecta a los diferentes grupos sociales y de qué manera esta circunstancia puede ser observada a través del voto, serán cuestiones esenciales de nuestros análisis.

A lo largo de nuestro trabajo de búsqueda de datos electorales, hemos atravesado tres fases: los correspondientes a los primeros comicios de 1990 fueron particularmente difíciles de obtener. En aquellos momentos, los resultados no se recogían en ninguna publicación oficial, y fue gracias al profesor de Ciencia Política y miembro de la Comisión Electoral Central Dimitar Dimitrov que pudimos acceder a la información necesaria. Después de estas primeras elecciones, los datos se ofrecían a través de una publicación oficial (Izbori Bulletin) en papel impreso. Y, en los últimos años, se puede acceder a los datos también a través de Internet, en las páginas web de la Comisión Electoral Central y de la Academia Búlgara de Ciencias.

\section{El contexto socioeconómico: vivir en tiempos de cambio}

Un aspecto relevante de cara a entender el comportamiento electoral de los ciudadanos búlgaros durante el periodo 1989-2009 es el rol cambiante de la política en la búsqueda de su bienestar social. El profundo deterioro de las 
condiciones de vida aparece, en todo caso, ligado a los devenires políticos y electorales, pero en Bulgaria encontramos hechos especialmente preocupantes. Datos del Eurobarometer de la Comisión Europea - procedentes de un trabajo de campo llevado a cabo en los países candidatos ${ }^{1}$ en octubre de $2001-$ muestran como latía el pulso vital de Bulgaria. Esta información tiene el valor añadido de su carácter comparativo, que acentúa el dramatismo de la situación búlgara y se refiere a aspectos de la vida cotidiana en los que puede ser más fácil encontrar el rostro humano de la crisis. Así, a la pregunta «Si compara su situación actual con la de hace cinco años, ¿diría que ha mejorado, que ha permanecido más o menos igual o que ha empeorado?», solo un 14 por ciento de los entrevistados búlgaros eligieron la primera opción, muy por debajo de la media (27 por ciento) y, ciertamente, con el menor porcentaje de todos los países analizados en la encuesta (European Commission, 2002: B-12). En lo referente a bienes materiales, a la pregunta de respuesta múltiple «¿Tiene usted acceso o usa... (un reproductor de vídeo, un fax, una antena parabólica, una televisión con teletexto, un teléfono móvil, un ordenador, un CD-ROM, un lector de CDI, un módem, Internet o ninguno de los anteriores?», los entrevistados búlgaros dieron respuestas positivas inferiores a la media en todos los casos, y un 51 por ciento de ellos contestaron «ninguno de los anteriores». Este porcentaje era el más elevado de todos los países candidatos y se situaba 35 puntos por encima de la media (ibídem: B-17). Muy significativamente, en 2007, el ingreso medio per cápita por hogar fue sólo de 3.347 levas (alrededor de 1.709 euros) (Statistical Reference Book of the Republic of Bulgaria 2008. Sofia: National Statistical Institute, p. 71).

¿Qué es tan distintivo de Bulgaria, que hace que sus gentes vivan de una manera tan particularmente sacrificada? Sintéticamente, podemos señalar lo que llamaríamos un factor inespecífico — común a todos los países del area- y uno específico - especialmente característico de Bulgaria. El inespecífico es lo que Luis Ângel Rojo expresaba, con referencia genérica a las extintas economías socialistas, con las palabras «sin plan y sin mercado»: «la disciplina impuesta por la planificación y la coerción, como principios ordenadores de la actividad económica, se ha visto debilitada sin que la sustituyesen nuevos elementos de disciplina resultantes de un funcionamiento real de los mercados. El resultado ha sido un considerable grado de caos» (Rojo, 1990: 30). Esta afirmación tan sumamente clarificadora todavía mantiene su vigencia en el caso búlgaro.

Otro factor relevante, de carácter específico, es el que señala Emil Giatzidis, para el que «en un país tan pequeño como Bulgaria, el comercio exterior es siempre de vital importancia para su desarrollo económico. Durante el comunismo, la economía era fuertemente dependiente del comercio exterior [...]. Con amplia diferencia, la mayor parte de este comercio [...] tenía lugar dentro del bloque comunista» (Giatzidis, 2002: 31-32). Durante el periodo de transición, como señala el profesor Francisco Veiga «colapsados los circuitos

1. Bulgaria, Chipre, República Checa, Estonia, Hungría, Lituania, Letonia, Malta, Polonia, Rumanía, Eslovaquia, Eslovenia y Turquía. 
Tabla 1. Pobreza en función del nivel educativo y el lugar de residencia

\begin{tabular}{|c|c|c|c|c|c|c|}
\hline \multirow[b]{2}{*}{ Nivel educativo } & \multicolumn{3}{|c|}{ Tasa de pobreza (\%) } & \multicolumn{3}{|c|}{ Nivel de pobreza (\%) } \\
\hline & Ciudad & Pueblo & Nacional & Ciudad & Pueblo & Naciona \\
\hline Primario o inferior & 25,7 & 30,7 & 28,0 & 7,7 & 8,1 & 7,9 \\
\hline Básico & 19,2 & 17,2 & 18,3 & 4,8 & 3,6 & 4,2 \\
\hline Secundario & 10,0 & 4,6 & 8,6 & 1,5 & 0,6 & 1,3 \\
\hline Secundario profesional & 6,1 & 6,2 & 6,1 & 1,4 & 1,5 & 1,4 \\
\hline Universitario & 3,3 & 7,1 & 3,7 & 0,6 & 1,5 & 0,7 \\
\hline
\end{tabular}

Tasa de pobreza: porcentaje de hogares pobres sobre el total de entrevistados.

Nivel de pobreza: ingreso medio relativo de hogares situados por debajo de la línea de pobreza.

Fuente: VVAA, 2003: 174

económicos del Comecon, pocas posibilidades tenían los búlgaros de colocar en el mercado internacional sus productos. La situación no habría sido tan dramática para Rumanía, debido a que ese país se había ido abriendo paso en mercados asiáticos y africanos que le aseguraron salidas comerciales incluso tras la caída del régimen comunista. Aunque los búlgaros también habían hecho sus pinitos en ese terreno, dependían más del comercio con la URSS y los países de la Europa Central» (Veiga, 1998: 69. En el mismo sentido, puede verse McIntyre, 1988: 171).

La pobreza que se derivaba de esta situación afectaba de manera diferente a la diversidad de grupos existentes en la población búlgara. Las diferencias aparecían de una manera especialmente visible sobre la base del nivel educativo y del tipo de hábitat. En este sentido, es importante poner de relieve que «el análisis de la pobreza por nivel educativo muestra una correlación nítida: a medida que el nivel educativo aumenta, la pobreza disminuye. El porcentaje relativo de pobres es más elevado entre la gente con educación primaria o inferior (28\%), que se sitúa en más del doble de la media nacional» (VVAA, 2003: 77). De la misma manera, pueden observarse diferencias significativas en lo que se refiere al lugar de residencia, como vemos en la tabla 1.

En consecuencia, los efectos de la crisis - tanto en el sentido económico como en el electoral - no aparecen distribuidos de una manera equitativa entre el conjunto de la población —ni tampoco las motivaciones electorales, como tendremos ocasión de ver. Cleavages como nivel educativo y tipo de residencia (hábitat) resultarán ser indicadores muy útiles para analizar la realidad electoral de la sociedad búlgara, en un contexto en el que la continuidad de la crisis es un elemento clave del comportamiento electoral de los ciudadanos de Bulgaria, como veremos en el siguiente epígrafe.

\section{El devenir de los procesos electorales: el voto en un entorno de crisis política y económica}

\subsection{El comienzo de la vida electoral democrática}

El sistema electoral utilizado en las primeras elecciones democráticas (junio de 1990) estuvo en vigor sólo durante este proceso. Los diputados 
electos acordaron establecer nuevas reglas para las elecciones posteriores. En 1990, se eligieron 200 escaños por sistema proporcional (mediante la formula D’Hondt), mientras otros 200 serían elegidos en circunscripciones uninominales sobre la base de un sistema mayoritario a dos vueltas. El ganador debería obtener más del 50 por ciento de los sufragios en la primera vuelta; si no era así, o si la participación era inferior a ese mismo porcentaje, tendría lugar una segunda vuelta con solo los dos candidatos más votados. En la parte elegida de manera proporcional, los escaños eran asignados a nivel nacional; una vez que, mediante la fórmula D'Hondt, se determinaba el número de escaños plurinominales que correspondía a cada partido o coalición, la asignación individual se llevaba a cabo por distritos, de nuevo mediante la fórmula D'Hondt. Sin la parte elegida por sistema mayoritario, el sistema electoral era muy altamente proporcional, debido a la gran magnitud de la circunscripción (todo el territorio de Bulgaria) utilizada para la distribución de escaños por candidaturas. La proporcionalidad sólo quedaba limitada por el mínimo del 4 por ciento de los votos necesario para tomar parte en la asignación de escaños.

De este modo, en el contexto de optimismo generado a raíz del cambio político, los búlgaros elegían los días 10 y 17 de junio de 1990 a sus 400 diputados democráticos en las que serían las primeras elecciones libres desde 1931. De los 40 partidos y grupos que presentaron candidatos, solo 4 conseguirían un rol relevante en el recién nacido panorama político. Los dos principales contendientes eran los ex comunistas del Partido Socialista Búlgaro, de un lado, y la Unión de Fuerzas Democráticas — representativa de la oposición al antiguo régimen-, de otro. Junto a ellos, en las áreas de influencia de la minoría de etnia turca ${ }^{2}$, el Movimiento por los Derechos y las Libertades obtuvo una relevancia electoral especialmente significativa.

Fue, sin duda, la propia dinámica de la sociedad búlgara la que generó los resultados de estos primeros comicios, con una alta participación fruto de las expectativas despertadas por la nueva vida en libertad (tabla 2). Estos resultados fueron los únicos en el área que dieron la victoria a un antiguo partido comunista (tabla 3). El rechazo al régimen comunista era bajo, en comparación con los otros países de la Europa del Este. La transición búlgara había sido relativamente pacífica, especialmente si la comparamos con el resto de países balcánicos; el trágico fin de Ceaucescu y el triste devenir de los acontecimientos en la ex Yugoslavia ofrecen contrapuntos apropiados para comprender esta afirmación en todo su dramatismo.

Las elecciones generales de 1991 — ya con 240 escaños elegidos únicamente mediante el mencionado sistema proporcional- representaron un cambio de gran magnitud. El Gobierno de la nación fue a manos de aquéllos que poco

2. Según los datos del censo de marzo de 2001 que recoge Yantsislav Yanakiev, los étnicamente búlgaros representan el 83,6\% de la población del país. Otros dos grupos étnicos, los turcos y los gitanos, representan, respectivamente, el 9,5\% y el 4,65\% del total poblacional. Otros grupos étnicos menores representan un 1,5\% (Yanakiev, 2008: 205). 
Tabla 2. Elecciones legislativas 1990. Datos globales

\begin{tabular}{lr}
\hline Parte proporcional & \\
\hline Distritos & 28 \\
Número total de papeletas & 6.333 .334 \\
Votos válidos & 6.124 .501 \\
\% participación & 90,60 \\
\hline Parte mayoritaria & \\
\hline Distritos & 200 \\
Primera vuelta & \\
Votos válidos & 6.334 .415 \\
En blanco y nulos & 244.296 \\
\% participación & 90,79 \\
Escaños elegidos & 119 \\
Segunda vuelta & \\
Votos válidos & 2.370 .812 \\
En blanco y nulos & 21.528 \\
\% participación & 84,14 \\
Escaños elegidos & 81 \\
\hline
\end{tabular}

Fuente: datos facilitados por el profesor Dimitar Dimitrov.

Tabla 3. Elecciones a la Gran Asamblea Nacional 1990

\begin{tabular}{|c|c|c|c|c|c|c|c|c|c|c|}
\hline & \multicolumn{4}{|c|}{ Parte proporcional } & \multicolumn{4}{|c|}{ Escaños uninominales } & \multicolumn{2}{|c|}{ Total de escaños } \\
\hline & Votos & $\%$ & Escaños & $\%$ & 10 junio & 17 junio & Total & $\%$ & & $\%$ \\
\hline PSB & 2.887 .677 & 47,15 & 97 & 48,5 & 75 & 39 & 114 & 57 & 211 & 52,75 \\
\hline UFD & 2.317 .698 & 36,20 & 75 & 37,5 & 32 & 37 & 69 & 34,5 & 144 & 36 \\
\hline MDL & 368.929 & 6,03 & 12 & 6 & 9 & 2 & 11 & 5,5 & 23 & 5,75 \\
\hline UNAB & 491.597 & 8,03 & 16 & 8 & 0 & 0 & 0 & 0 & 16 & 4 \\
\hline PPT & 36.668 & 0,6 & 0 & 0 & 0 & 1 & 1 & 0,5 & 1 & 0,25 \\
\hline PSD & 3.036 & 0,05 & 0 & 0 & 1 & 0 & 1 & 0,5 & 1 & 0,25 \\
\hline PAS & 22.064 & 0,3 & 0 & 0 & 0 & 0 & 0 & 0 & 0 & 0 \\
\hline Indep. & - & - & - & - & 2 & 2 & 4 & 2 & 4 & 1 \\
\hline
\end{tabular}

Fuente: Comisión Electoral Central y profesor Dimitar Dimitrov.

tiempo antes representaban ideologías rigurosamente prohibidas por la ley. Y, junto a ello, la estabilidad del país dependía del partido representativo de la minoría de etnia turca. Los «temidos» turcos, que sufrieron durante el régimen comunista duros procesos de asimilación, resultaban ser ahora decisivos en el mapa político búlgaro.

A las elecciones de 13 de octubre de 1991 concurrieron 38 listas que representaban a un partido o coalición y 19 candidaturas independientes ${ }^{3}$. Según los

3. Biuletin Izbori 91. Sofia: Tsentralna Izviratelna Komissia, p. 13. 
Tabla 4. Elecciones legislativas 1991. Resultados electorales

Electores registrados 6.790.006

Votantes 5.694.842

\% participación $83,87 \%$

\begin{tabular}{lcrcc}
\hline Partidos y coaliciones & \multicolumn{1}{c}{ Votos } & \multicolumn{1}{c}{$\%$} & Escaños & $\%$ \\
\hline PSB & 1.836 .050 & 33,14 & 106 & 44,20 \\
UFD & 1.903 .567 & 34,36 & 110 & 45,80 \\
MDL & 418.168 & 7,55 & 24 & 10,00 \\
Otros & 1.282 .052 & 24,95 & & \\
Total & 5.540 .837 & 100,00 & 240 & 100,00 \\
\hline
\end{tabular}

Fuente: Biuletin Izbori '91. Sofia: Tsentralna Izbiratelna Komissia, p. 12-13.

datos proclamados oficialmente, la participación en estas segundas elecciones parlamentarias fue también alta ( 83,87 por ciento), aunque no tanto como en las anteriores. El resultado redujo significativamente la diferencia entre las dos primeras opciones, con la inversión de papeles de primera y segunda fuerza. La Unión de Fuerzas Democráticas consiguió el 34,36 por ciento de los votos válidos - sólo 1,22 puntos por encima del Partido Socialista. Como consecuencia, el Movimiento por los Derechos y las Libertades asumió el papel de partido bisagra, con una alta capacidad de influencia en la vida política búlgara. Ninguno de los candidatos independientes alcanzó los 12.742 votos necesarios para superar el mínimo legal.

Desde los primeros momentos de la transición, los puntos de vista de la población acerca del cambio quedaban claramente definidos en función de los distintos grupos sociodemográficos — con su traducción en el comportamiento electoral. Concretamente, las actitudes hacia algo tan cargado de significación como el proceso de privatización quedaban bien definidas en aquellos momentos iniciales, y eran un buen reflejo de los sentimientos y las percepciones que tenían los distintos sectores. Como sintetiza un informe del Center for the Study of Democracy:

Los sectores de población de mayor nivel educativo y de cualificación son los primeros en percibir la necesidad de cambios radicales. Muchos de ellos trabajan para empresas estatales y para las instituciones, y viven en la capital y en las grandes ciudades. Son principalmente gente joven (de entre 29 y 40 años), predominantemente varones y partidarios de la UFD. Entre los que consideran que la privatización es indispensable y que debería ser llevada a cabo en el menor tiempo posible, casi el 50\% tiene estudios universitarios, y el $40 \%$ tiene educación secundaria especializada. El 51\% apoya a la UFD (el doble de los que apoyan al PSB). [...]

Los resultados de la encuesta muestran que los que se oponen a la privatización son principalmente pensionistas, trabajadores agrícolas y desempleados. Se trata de gente de menor nivel educativo, de mayor edad (ocupan el 
mayor rango de la escala de edad), viven mayoritariamente en las ciudades más pequeñas y en los pueblos, son predominantemente mujeres, muchos de ellos desempleados y, por lo tanto, con bajos niveles de vida. Su inestable situación económica les genera una disposición negativa a los cambios radicales, incluyendo la privatización. La mayoría de ellos apoya al PSB ${ }^{4}$. (Center for the Study of Democracy, 1992)

Las elecciones legislativas de 1991 y las presidenciales de 1992 muestran cierta continuidad en los resultados, en la medida en que, como consecuencia de ambas, figuras representativas de la oposición al régimen comunista alcanzaron el poder. En 1992, resultó especialmente interesante observar el apoyo real que podía conseguir en las urnas el líder más emblemático del movimiento anticomunista. Zheliu Zhelev concurría ostentando la presidencia de la República, otorgada por elección del Parlamento el 1 de agosto de 1990. El Partido Socialista no propuso un candidato propio y expresó su apoyo al abogado Velko Vulkanov. Concurrieron otras 19 candidaturas, con pocas opciones de alcanzar la segunda vuelta. Entre ellas, estaba la del excéntrico millonario Georges Ganchev, que lideraba el Bloque Búlgaro de Negocios de una manera un tanto histriónica. La primera vuelta tuvo lugar el 12 de enero de 1992, con algunos resultados esperados - la presencia de Zhelev y Vulkanov en la segunda vuelta - y otros inesperados — como el elevado apoyo que consiguió Ganchev.

La normativa electoral establecía dos requisitos que, incluso con la confluencia de sólo uno de ellos, harían necesaria la segunda vuelta: si ningún candidato obtenía más del 50 por ciento de los votos, o si la participación no alcanzaba esa cifra, se habría de llevar a cabo la segunda ronda de votaciones. En este caso, se dio el primer requisito. Se esperaba que la segunda vuelta ofreciera una participación mucho más elevada, como consecuencia de la competencia entre las dos «culturas» del país y el carácter definitivo de los resultados. Sin embargo, sólo participaron 66.335 votantes más en la segunda ronda, con un incremento de sólo 0,51 puntos. Lógicamente, las dos candidaturas consiguieron más votos en la segunda vuelta, pero en mayor medida la respaldada por el PSB. La candidatura de Zhelev obtuvo 464.869 votos más, mientras que la de Vulkanov recogió un incremento de 893.464. En porcentajes, la ventaja de 14 puntos de la primera vuelta quedó reducida a 5,7 en la segunda.

Los resultados ponían de manifiesto la consistencia de las dos principales opciones políticas. En un contexto muy propicio para Zhelev y la UFD, la candidatura que representaba las posiciones socialistas mostraba una noto-

4. También la profesora Petya Pachkova ponía de relieve la relevancia de las diferencias entre rural y urbano para una adecuada comprensión del comportamiento electoral de los búlgaros. Concretamente, señala los recuerdos de la vida tranquila y plácida de los años de comunismo como un factor que contribuiría a explicar el apoyo a los socialistas en las áreas rurales; por el contrario, algunas propuestas de la UFD — consideradas radicales por algunos habitantes de zonas rurales - generaban en ciertos sectores de población no urbana un temor hacia las reformas rápidas (entrevista con Petya Pachkova, Sofia, 23 de septiembre de 1998). 
Tabla 5. Elecciones presidenciales 1992. Resultados electorales. Primera vuelta

\begin{tabular}{lrr}
\hline $\begin{array}{l}\text { Electores registrados } 6.817 .914 \\
\text { Votantes 5.139.891 }\end{array}$ & \\
Participación 75,39\% & \multicolumn{1}{c}{ Votos } & $\%$ \\
\hline Candidatos & 2.273 .541 & 44,66 \\
\hline Zheliu Zhelev/Blaga Dimitrova & 113.897 & 2,24 \\
Blagovest Sendov/Ognian Saparev & 1.549 .970 & 30,44 \\
Velko Vulkanov/Rumen Vodenicharov & 854.108 & 16,78 \\
Georgi Ganchev/Petar Berov & 299.611 & 5,87 \\
Otros &
\end{tabular}

Fuente: Biuletin Prezidentski Izbori '92. Sofia: Tsentralna Izbiratelna Komissia, p. 7 y 14.

Tabla 6. Elecciones presidenciales 1992. Resultados electorales. Segunda vuelta

\begin{tabular}{lcc}
\hline Electores registrados 6.859 .318 & & \\
Votantes 5.206 .226 & & \\
Participación $75,90 \%$ & Votos & $\%$ \\
\hline Candidatos & 2.738 .420 & 52,85 \\
\hline Zheliu Zhelev/Blaga Dimitrova & 2.443 .434 & 47,15 \\
\hline
\end{tabular}

Fuente: Biuletin Prezidentski Izbori '92. Sofia: Tsentralna Izbiratelna Komissia, p. 7 y 14.

ria solidez - especialmente teniendo en cuenta la poderosa figura pública de Zheliu Zhelev. Los datos permitían vislumbrar un reducto de voto socialista de alto nivel de fidelidad.

\subsection{La relevancia de la economía y la vida cotidiana}

$\mathrm{Si}$, en 1990, los programas electorales estaban centrados en los problemas de la democratización, en 1994 el debate giró en torno a los aspectos económicos. Se esperaba que los comicios de 1994 dieran una respuesta a las demandas de orden social y estabilidad que surgían de la ya sufrida población búlgara. Los precios se habían incrementado un 59,9 por ciento entre enero y junio de 1994. En este año electoral, el producto interior bruto se había reducido una cuarta parte en relación con 1990; la producción industrial había caído alrededor del 50 por ciento (Genov, 1995: IX). En expresión del informe Bulgaria. Human Development Report 1995, «en algunos momentos, la economía búlgara parecía casi inmanejable» (ibídem: 4).

Finalmente, es la UFD la que sufre en mayor medida la erosión derivada de haber sido la referencia del electorado en sus expectativas de una vida mejor. La Unión de Fuerzas Democráticas — o lo que todavía quedaba de ella - perdió en estos comicios 650.000 votos respecto a 1991, y alrededor de 1.000 .000 respecto a 1990. En los resultados finales, 125 de los 240 escaños en liza fueron 
Tabla 7. Elecciones legislativas 1994. Resultados electorales

Votantes censados 6.987 .645

Votantes 5.264.448

Participación $75,23 \%$

\begin{tabular}{lrrrr}
\hline Partidos y coaliciones & \multicolumn{1}{c}{ Votos } & \multicolumn{1}{c}{$\%$} & Escaños & \multicolumn{1}{c}{$\%$} \\
\hline PSB/UNAB A. S./Ecoglasnost & 2.262 .943 & 43,50 & 125 & 52,08 \\
Unión de Fuerzas Democráticas & 1.260 .374 & 24,23 & 69 & 28,75 \\
Unión Popular & 338.478 & 6,51 & 18 & 7,50 \\
Movimiento por los Derechos y las Libertades & 283.094 & 5,44 & 15 & 6,25 \\
Bloque Búlgaro de Negocios & 245.849 & 4,73 & 13 & 5,42 \\
Otros & 811.327 & 15,60 & & \\
Total & 5.202 .065 & 100,00 & 240 & 100,00 \\
\hline
\end{tabular}

Fuente: Biuletin Izbori '94. Sofia: Tsentralna Izbiratelna Komissia, p. 12-14.

Tabla 8. Elecciones presidenciales 1996. Resultados electorales. Primera vuelta

Votantes censados 6.822.045

Votantes 4.317.161

Participación 63,14\%

\begin{tabular}{lrr}
\hline Candidatos & \multicolumn{1}{c}{ Votos } & $\%$ \\
\hline Boichev/Kulekov & 57.668 & 1,34 \\
Marazov/Bokova & 1.158 .204 & 27,01 \\
P. Stoyanov/Kavalkjiev & 1.889 .825 & 44,07 \\
Tomov/Marinchevski & 135.571 & 3,16 \\
Ganchev/Antonov & 937.686 & 21,87 \\
Otros & 109.546 & 2,55 \\
Total & 4.288 .500 & 100,00 \\
\hline
\end{tabular}

Fuente: Biuletin Rezultatite ot Izborite na President $i$ Vitsepresident na Republicata. Proizbedeni na 27 Octombri y 3 Noembri 1996 Godina. Sofia: Tsentralna Izbiratelna Komisia, p. 1-2.

Tabla 9. Elecciones presidenciales 1996. Resultados electorales. Segunda vuelta

Votantes censados 6.820.913

Votantes 4.215.145

Participación 61,67\%

\begin{tabular}{lcr}
\hline Candidatos & Votos & $\%$ \\
\hline Marazov/Bokova & 1.687 .242 & 40,27 \\
Stoyanov/Kavalkjiev & 2.502 .517 & 59,73 \\
Total & 4.189 .759 & 100,00 \\
\hline
\end{tabular}

Fuente: Biuletin Rezultatite ot Izborite na President i Vitsepresident na Republicata. Proizbedeni na 27 Octombri y 3 Noembri 1996 Godina. Sofia: Tsentralna Izbiratelna Komisia, p. 1-2. 
para la coalición liderada por el Partido Socialista Búlgaro, y aproximadamente la mitad para los ganadores de 1991 (tabla 7). Ello nos da una muestra de los devastadores efectos de la crisis económica. En las siguientes elecciones, este factor se verá amplificado. El acceso al Parlamento por parte de dos nuevos partidos es un claro indicativo del hecho de que la victoria socialista era - al menos parcialmente - el resultado del deterioro de la UFD, lo que traería, como consecuencia adicional, la división interna de esta opción — con el consiguiente coste electoral.

En las elecciones presidenciales de 27 de octubre y 3 de noviembre de 1996, se mantiene la misma tónica de alternancia ya observada en comicios anteriores. Sin embargo, en este caso, tiene lugar de una manera peculiar: por medio de elecciones primarias en las que, a la manera americana, se permite la participación de los no afiliados. Las elecciones primarias se celebran, según algunos observadores, como iniciativa del presidente Zheliu Zhelev. Según estas fuentes, Zhelev no confiaba en ser elegido como candidato de la UFD y pensó que, a través del voto popular, su nominación sería más probable. Sus previsiones resultaron, finalmente, frustradas, y el abogado Petar Stoyanov consiguió la nominación.

Desde mayo de 1996, la población estaba sufriendo un agudo deterioro de la calidad de vida, como consecuencia de la devaluación de la moneda búlgara (el leva) y la alta inflación que conllevó. Algunos meses después, tuvo lugar el conocido asalto al Parlamento por parte de grupos de manifestantes. El contexto de las elecciones presidenciales de octubre de 1996 era, pues, de abierto rechazo al polo socialista, al que se hacía responsable de la lamentable situación del país. La primera, y esperada, consecuencia fue la elección del representante de la oposición al polo socialista. Además, la generalizada situación de desencanto dio como resultado un apoyo extremadamente elevado a Georges Ganchev, con unos niveles de voto muy cercanos a los de los socialistas que casi rompen la tendencia bipolar de la vida política búlgara.

La polarización de la segunda vuelta favoreció a los candidatos socialistas. Es razonable pensar que buena parte de los votos de Ganchev fueron a los candidatos respaldados por el PSB. El componente populista y nacionalista de la opción representada por Ganchev nos da la clave de la cercanía entre ambos electorados.

\subsection{Cambio en el mapa político: la aparición exitosa de nuevas opciones}

El dramático contexto de las elecciones presidenciales de 1996 todavía existía en abril del año siguiente, cuando tienen lugar las elecciones generales. En 1996, la inflación alcanzó el 311,1 por ciento. Estas aterradoras cifras todavía se verían sobrepasadas en 1997, especialmente en los primeros meses del año, y finalmente llegarían a alcanzar el 578,7 por ciento. Como consecuencia de esta triste realidad, tuvo lugar el ya mencionado asalto al edificio de la Asamblea Nacional. Poco después, el Parlamento es disuelto y se convocan elecciones anticipadas. 
En 1997, la coalición originariamente denominada Unión de Fuerzas Democráticas se había transformado en un partido político que, a su vez, lideraba una coalición llamada Fuerzas Democráticas Unidas ${ }^{5}$. Estaba integrada, además, por el Partido Democrático, la Unión Nacional Agraria Búlgara y el Partido Socialdemócrata ${ }^{6}$. El Partido Socialista Búlgaro sobrevivió electoralmente bajo la forma de Izquierda Democrática, después de algunas escisiones. El Movimiento por los Derechos y las Libertades lideró la Unión por la Salvación Nacional, tratando de ocupar el espacio político de centro, al que también optaba Euroizquierda (integrada por antiguos miembros del Partido Socialista). El Bloque Búlgaro de Negocios de Georges Ganchev adoptó una estrategia y una actitud que fue considerada de centro nacionalista.

En este contexto, se esperaba una amplia victoria electoral de la oposición. La consistencia socialista ante las urnas representó una sorpresa, con un 22,07 por ciento de los votos recogidos en unas circunstancias sumamente adversas. La polarización de la vida política búlgara todavía latía en las cifras electorales. La traducción que el sistema electoral hacía de la voluntad popular ofrecía una mínima desviación: todos los partidos o coaliciones que superaron el mínimo establecido y consiguieron representación parlamentaria recibieron un pequeño bonus. Los porcentajes de escaños eran ligeramente superiores a los de votos (tabla 10).

En pocas palabras, como sintetiza el sociólogo Boris Gurov, «la historia del periodo 1990-1997 es la historia de las reformas fracasadas», lo que generó un persistente «voto de protesta». Después de esos años, aparece un periodo nuevo cuyo elemento definitorio esencial es, para el profesor Gurov, el «cambio del voto de partido al voto en función de la persona» ${ }^{7}$. Los liderazgos pasan a ocupar, en mayor medida, el centro de atención. De alguna manera, parece como si la búsqueda de soluciones basadas en ideologías dejara paso a una búsqueda de personalidades presuntamente capaces de acabar con las dificultades económicas y la corrupción. Como consecuencia, emergieron nuevas opciones políticas — difícilmente encuadrables en partidos tradicionales-, encabezadas por figuras políticas igualmente novedosas. Durante esta etapa, estas opciones conseguirían amplias victorias electorales —algo que no ocurrió

5. En búlgaro, la diferencia nominal es más clara: Saiuz na Demokratichnite Sili cambia a Obedineni Demokratichni Sili.

6. La representación de la internacionalmente poderosa socialdemocracia muestra alguna peculiaridad en Bulgaria, principalmente porque, debido al pequeño tamaño y a la escasa influencia de los grupos que reclamaban esa denominación, fue el PSB el que finalmente logró aglutinar esa sensibilidad política. Recordemos que esta opción fue aceptada en la Internacional Socialista. En palabras de la socióloga búlgara Boriana Dimitrova, «detrás del término socialdemocracia se ocultaban muchos intereses y proyectos políticos personales [...]. Sería posible hablar de una base electoral socialdemócrata diferenciada sólo en el caso de que las respectivas opciones encontraran un cauce común de consolidación con las personas adecuadas y con comportamientos y valores compartidos» (Dimitrova, 1997: 60). Esta situación, en mi opinión, no ha tenido lugar.

7. Entrevista con Boris Gunov, sociólogo del Institute of Sociology of Bulgarian Academy of Sciences (Sofia, 26 de mayo de 2010). 
Tabla 10. Elecciones parlamentarias 1997. Resultados electorales

Votantes censados 6.819 .511

Votantes 4.191.257

Participación 58,86\%

\begin{tabular}{lrrrr}
\hline Candidaturas & \multicolumn{1}{c}{ Votos } & \multicolumn{1}{c}{$\%$} & Escaños & $\%$ \\
\hline Bloque Búlgaro de Negocios & 209.796 & 4,93 & 12 & 5,00 \\
Fuerzas Democráticas Unidas & 2.223 .714 & 52,26 & 137 & 57,08 \\
Euroizquierda & 234.058 & 5,50 & 14 & 5,83 \\
Izquierda Democrática & 939.308 & 22,07 & 58 & 24,17 \\
Unión para la Salvación Nacional & 323.429 & 7,60 & 19 & 100,00 \\
Total & 3.930 .305 & 92,36 & 240 & 100,00 \\
\hline
\end{tabular}

Fuente: Biuletin za Rezultatite ot Izborite. Proizbedeni na 19 April 1997 G.. Sofia: Tsentralna Izbiratelna Komisia, p. 6 and VII. La cifra de participación se calcula a partir de los datos recogidos en p. 5 y V.

con partidos que anteriormente habían estado basados en liderazgos personales, como el Bloque Búlgaro de Negocios.

En este sentido, las elecciones parlamentarias de junio de 2001 representaron el inicio de una nueva etapa en el panorama político y social de Bulgaria, con connotaciones que transcendían los límites de esta frecuentemente desconocida República de los Balcanes. Desde 1989, su desarrollo político había estado definido por un continuo movimiento de péndulo, como consecuencia de la persistente desesperanza de la población búlgara. Entre 1997 y 2001, los indicadores estadísticos parecían apuntar hacia una atenuación de la desazón que subyacía a cada uno de los resultados electorales del periodo democrático. La inflación, que alcanzó el 578,7 por ciento en 1997, era de sólo el 0,3 en 1999, y de 9,9 en 2000. Este dato, tan sensible para la vida cotidiana - y, por lo tanto, para el voto- aparecía acompañado por una favorable evolución de la tasa de cambio con el dólar. La estabilidad en los datos macroeconómicos básicos llevó al primer ministro Ivan Kostov a afirmar, en una reunión del Partido Popular Europeo celebrada en Sofía el 5 de abril de 2001, que Bulgaria alcanzaría en breve el pleno empleo siguiendo las políticas del presidente Aznar. La legislatura comprendida entre 1997 y 2001 era la primera que concluía sin convocatoria de elecciones anticipadas. Las candidaturas búlgaras para la integración en la Unión Europea y en la Alianza Atlántica parecían estar bien encaminadas.

Sólo dos meses antes de las elecciones de junio, los sondeos otorgaban entre un 25 y un 30 por ciento de los votos a la opción liderada por el ex zar Simeón II, lo que le situaba como tercera fuerza — probablemente incluso la segunda—, muy lejos del tremendo impacto electoral que finalmente habría de representar. Con todo, las dificultades cotidianas subsistían para la inmensa mayoría de la población —que se mostraba especialmente sensible ante la incapacidad del Gobierno para controlar la corrupción-, y los mecanismos de mercado no conseguían arraigar en la vida democrática búlgara. La figura predominante en 
Tabla 11. Elecciones parlamentarias 2001. Resultados electorales

\begin{tabular}{|c|c|c|c|c|}
\hline \multicolumn{5}{|l|}{ Votantes censados 6.874 .668} \\
\hline Votantes 4.608.135 & & & & \\
\hline \multicolumn{5}{|l|}{ Participación 67,03\% } \\
\hline Partido o coalición & Votos & $\%$ votos & Escaños & \% escaños \\
\hline Fuerzas Democráticas Unidas & 830.338 & 18,18 & 51 & 21,25 \\
\hline Coalición por Bulgaria (PSB) & 783.872 & 17,15 & 48 & 50,00 \\
\hline Movimiento Nacional Simeón II & 1.952 .513 & 42,74 & 120 & 20,00 \\
\hline MDL-Unión Liberal-Euroroma & 340.395 & 7,45 & 21 & 8,75 \\
\hline Gergyovden-VMRO & 165.927 & 3,63 & - & - \\
\hline Coalición Simeón II & 157.141 & 3,44 & - & - \\
\hline Otros & 338.505 & 7,41 & 0 & 0 \\
\hline Total & 4.568.191 & 100,00 & 240 & 100,00 \\
\hline
\end{tabular}

Fuente: Central Electoral Commission of the Republic of Bulgaria (http://www.math.bas.bg/izbori/res/2001/ kpe00.htm).

la opinión pública búlgara era Simeón Sajonia-Coburgo. El incremento en los sondeos era continuado, y finalmente su opción resultó claramente victoriosa en las urnas. El electorado puso sus esperanzas en una opción que evocaba la idea de otra forma diferente de política y de gobierno - aunque no fueran explícitamente formuladas como tales. Para muchos electores, el rey que vino de Occidente era, básicamente, una nueva esperanza, aparentemente sólida, que no había sufrido la erosión del tortuoso proceso de cambio búlgaro.

En la configuración parlamentaria de 2001, subsistían los más clásicos representantes del mapa de partidos búlgaro, que habían protagonizado los primeros años del actual periodo democrático y que concurrían en coaliciones en las que desempeñaban un rol claramente protagonista y dominante. El papel estelar, sin embargo, correspondía sin duda a la opción encabezada por Simeón SajoniaCoburgo. No se trataba sólo de que fuera la opción ganadora, sino que además se daba la circunstancia de que estaba liderada por una figura especialmente característica, que dejó el país con solo nueve años de edad y que ahora conseguía recoger las esperanzas de sus compatriotas. La situación era, por lo tanto, atípica, y logró sorprender a todo tipo de analistas. Si tratamos de ubicar el Movimiento Nacional Simeón II en el marco de los cleavages que han venido caracterizando a las opciones políticas búlgaras, tendríamos, como es lógico, unas dificultades algo mayores de lo habitual. Tomando en consideración el dualismo eslavo/occidental, la figura de Simeón Sajonia-Coburgo podría localizarse en el segundo polo, dadas sus connotaciones profesionales y personales y, ciertamente, sus posiciones políticas. Un segundo eje básico sería el estatistas/ liberales, sobre cuya base este movimiento podría clasificarse en el segundo polo.

El bipartidismo que ha caracterizado el actual periodo democrático búlgaro - con la concreción que siempre precisa la presencia del Movimiento por los Derechos y las Libertades — queda completamente desfigurado después de las 
Tabla 12. Elecciones presidenciales 2001. Resultados electorales. Primera vuelta

\begin{tabular}{lrr}
\hline $\begin{array}{l}\text { Votantes inscritos 6.824.979 } \begin{array}{l}\text { Votos válidos } 2.850 .291 \\
\text { Participación } 41,76 \%\end{array} \\
\text { Candidaturas }\end{array}$ & \multicolumn{1}{l}{ Votos } & \% votos \\
\hline Petar Beron & 31.394 & 1,11 \\
Petar Stoyanov & 991.680 & 34,95 \\
Bogomil Bonev & 546.801 & 19,27 \\
Zhorzh Ganchev & 95.481 & 3,36 \\
Georgi Parvanov & 1.032 .665 & 36,39 \\
Reneta Indzhova & 139.680 & 4,92 \\
Total & 2.837 .701 & 100,00
\end{tabular}

Fuente: Central Electoral Commission of the Republic of Bulgaria (http://www.math.bas.bg/izbori/ res/2001prezident/tur1/6kpe00.htm).

Tabla 13. Elecciones presidenciales 2001. Resultados electorales. Segunda vuelta

Votantes inscritos 6.868.407

Votos válidos 3.784 .033

Participación 55,09\%

\begin{tabular}{lcr}
\hline Candidaturas & Votos & \% votos \\
\hline Petar Stoyanov & 1.731 .676 & 45,87 \\
Georgi Parvanov & 2.043 .443 & 54,13 \\
Total & 3.775 .119 & 100,00 \\
\hline
\end{tabular}

Fuente: Central Electoral Commission of the Republic of Bulgaria (http://www.math.bas.bg/izbori/ res/2001 prezident/tur2/6kpe00.htm).

elecciones del 17 de junio de 2001. El efecto del sistema electoral es visible en lo que se refiere al mínimo del 4 por ciento necesario para tomar parte en el reparto de escaños, especialmente porque dos opciones consiguen dígitos muy cercanos al mínimo, sin sobrepasarlo. Teniendo en cuenta este hecho, la distribución de escaños a nivel nacional no genera sobrerrepresentaciones o distorsiones significativas, a pesar de que es llevada a cabo con la ley D'Hondt.

La desesperanza movilizada en las elecciones de junio de 2001, que a la postre otorgaría a Simeón Sajonia-Coburgo la jefatura del Gobierno, aparece de una manera tangible a través de otro dato igualmente cargado de significación. La participación había sido decreciente desde los primeros comicios de 1990 (con un 90,60 por ciento en la parte elegida de forma proporcional, recordemos). En 1997 llegó a alcanzar un reducido 58,86 por ciento. Es, sin duda, una cuantificación realmente expresiva del desafortunado desarrollo de la vida cotidiana en Bulgaria. La irrupción de una nueva oferta, a la que se otorga una apreciable solidez, permite — diríamos que de una manera también inesperada - invertir la línea continuamente decreciente. 
En pocas palabras, el año electoral 2001 será recordado, en lo que a Bulgaria se refiere, como aquel en el que los cleavages tradicionales de la vida política perdieron su centralidad. Sin embargo, la nueva figura protagonista del panorama político búlgaro no tomó parte en las elecciones presidenciales. La presencia de candidatos mantenía una apreciable similitud con anteriores comicios de este tipo. En el polo tradicionalmente ocupado por la denominada sensibilidad anticomunista, encontramos de nuevo al entonces presidente Petar Stoyanov, respaldado por la Unión de Fuerzas Democráticas y el Movimiento Nacional Simeón II; en el polo opuesto concurría, con el Partido Socialista como principal apoyo, su líder Georgi Parvanov. Junto a ellos, competía el peculiar Georges Ganchev, que también tomó parte en elecciones anteriores.

En contra de las previsiones de los sondeos, el ganador - tanto en la primera como en la segunda vuelta - fue el historiador de 44 años de edad Georgi Parvanov. Conviene tener en cuenta, dada la especial incidencia de los factores personales en las elecciones presidenciales, que el perfil político de Parvanov no era equiparable al de los líderes socialistas que le habían precedido, como Lukanov o Videnov. Sus esfuerzos para conducir a su partido hacia el espectro que pudiéramos llamar socialdemócrata rompió con líneas anteriores de mayor proximidad a las señas de identidad más clásicas del PSB. Esta línea política se concretaba en la siempre emblemática política exterior búlgara, cuya continuidad —incluida la adhesión a la Alianza Atlántica— garantizó inmediatamente después de su victoria electoral. Georgi Parvanov resultó ser, parafraseando un tanto espúriamente a Otto Kirchheimer ${ }^{8}$ (1996), un exitoso candidato catch-all.

Volvemos, una vez más, a la realidad de la desesperanza; y la encontramos, también de nuevo, a través de los correspondientes datos de participación. Con la excepción de las elecciones legislativas de 2001, las cifras de afluencia a las urnas han evolucionado de una manera continuamente decreciente, como reflejo del desasosiego con el que las gentes de Bulgaria afrontan sus vidas cotidianas.

La segunda vuelta de las elecciones presidenciales tuvo lugar el 18 de noviembre de 2001, porque en la primera ronda no se cumplieron ninguno de los requisitos legales establecidos. Los dos candidatos reproducían el cleavage ya clásico en el panorama político búlgaro; sin embargo, no ocurría lo mismo con el apoyo que ambos cosecharon en la ronda definitiva. El Movimiento por los Derechos y las Libertades, tradicional adversario de la sensibilidad socialista, expresó su apoyo público a Georgi Parvanov, y justificaba este posicionamiento en base a las expectativas que el candidato Petar Stoyanov - que, recordemos, ejercía la presidencia en el momento de estos comicios- había defraudado.

8. Como señala el profesor de Ciencia Política Dimitar Dimitrov (entrevista mantenida en Sofía el 29 de septiembre de 1998), en sentido estricto, «no es posible determinar si la tesis de Otto Kirchheimer puede ser considerada válida para la realidad búlgara, porque cada elección se ha celebrado en un contexto de crisis; no ha habido elecciones en condiciones de normalidad». Desde mi punto de vista, aunque la entrevista no es reciente, la opinión es perfectamente aplicable a la totalidad del periodo democrático. 
Tabla 14. Datos de opinión pública. «¿Cómo ve hoy la situación económica de su familia, comparada con la situación del año anterior?» Report 7, 2005

\begin{tabular}{|c|c|c|c|}
\hline & Ahora es mejor & Ahora es peor & No hay diferencia \\
\hline Total & 10 & 33 & 54 \\
\hline Varones & 12 & 31 & 54 \\
\hline Mujeres & 9 & 34 & 53 \\
\hline $18-30$ & 17 & 28 & 51 \\
\hline $31-40$ & 14 & 31 & 53 \\
\hline $41-50$ & 10 & 32 & 56 \\
\hline $51-60$ & 9 & 34 & 55 \\
\hline $61+$ & 5 & 37 & 54 \\
\hline PSB & 7 & 37 & 55 \\
\hline UFD & 14 & 26 & 58 \\
\hline MDL & 11 & 26 & 60 \\
\hline MNS-II & 25 & 13 & 60 \\
\hline No votan & 5 & 41 & 49 \\
\hline Búlgaros & 11 & 31 & 55 \\
\hline Turcos & 8 & 36 & 52 \\
\hline Gitanos & 4 & 51 & 38 \\
\hline Pueblos & 9 & 33 & 55 \\
\hline Ciudades pequeñas & 9 & 36 & 52 \\
\hline Capitales de distrito & 12 & 33 & 52 \\
\hline Sofia ciudad & 11 & 26 & 58 \\
\hline Trabajadores & 14 & 26 & 58 \\
\hline Jubilados & 6 & 37 & 54 \\
\hline Estudiantes & 22 & 18 & 52 \\
\hline Desempleados & 7 & 48 & 42 \\
\hline Empresarios & 23 & 20 & 54 \\
\hline Educación superior & 16 & 23 & 60 \\
\hline Educación secundaria & 13 & 31 & 54 \\
\hline Primaria o inferior & 5 & 39 & 50 \\
\hline Ingresos inferiores a $100 \mathrm{BGN}$ & 7 & 39 & 50 \\
\hline Ingresos entre 101 y 200 BGN & 11 & 30 & 57 \\
\hline Ingresos entre 201 y 300 BGN & 20 & 18 & 58 \\
\hline Ingresos superiores a $300 \mathrm{BGN}$ & 34 & 7 & 56 \\
\hline Izquierda & 7 & 39 & 53 \\
\hline Derecha & 14 & 27 & 58 \\
\hline Centro & 21 & 21 & 56 \\
\hline
\end{tabular}

Fuente: http://www.gallup-bbss.com/images/freeindex/free3.gif (consulta: 12 de mayo de 2010).

Los líderes del MDL ponían el énfasis en la necesidad de un presidente con una marcada orientación social. El resultado final resultó ser realmente inesperado, básicamente por dos razones: de un lado, las cifras de indecisos se situaron en torno a los veinte puntos; de otro lado, por la existencia de un voto oculto mayoritariamente socialista.

El contexto social de las elecciones parlamentarias de 2005 queda muy adecuadamente reflejado en la tabla 14 . No se trataba solo de que la gran 
mayoría de los entrevistados afirmaran que no percibían diferencias entre la situación económica de su familia en el momento de la encuesta y la del año anterior. Junto a ello, el 33 por ciento que contestaron que "Es ahora peor» dibujaba un panorama realmente preocupante. Dadas las elevadas cifras que encontramos en la columna "No hay diferencia», los dígitos en el resto de ellas resultan ser menos significativos; sin embargo, es posible identificar con facilidad los rasgos sociodemográficos que han caracterizado a la población de Bulgaria durante la transición, con unas percepciones más optimistas en los estratos de población de mayor nivel educativo, de menor nivel de edad y mejor condición socioeconómica, y con la población de características sociológicamente opuestas optando en mayor medida por la desesperanzadora opción «Ahora es peor». Las diferencias entre los votantes de las distintas opciones eran también visibles, y mostraban como los partidarios del MDL y el PSB estaban sufriendo en mayor medida las dificultades de la transición, algo que se aprecia muy nítidamente cuando comparamos sus actitudes con las de los votantes del MNS-II.

En lo que respecta a los resultados electorales, en las elecciones parlamentarias de 2005 encontramos dos características principales: en primer lugar, los resultados produjeron la Asamblea Nacional más fragmentada de todo el actual periodo democrático; en segundo lugar, la participación fue la más baja de las registradas en elecciones parlamentarias - algo que era, sin duda, otro claro indicador de la desafortunada evolución de la sociedad búlgara durante estos veinte años de vida democrática.

Una vez más, los resultados dieron lugar a un cambio institucional: la opción en la que tantas esperanzas se habían depositado -el Movimiento Nacional Simeón II - fue la clara perdedora. Las razones de este fracaso pueden hallarse en dos aspectos en los que se esperaba del ex zar una eficiencia especial. De un lado, la gestión de la crisis económica. Con el transcurrir del tiempo, se ponía de manifiesto que la solución a los problemas económicos no llegaba - con la decepción que ello acarreaba. De otro lado, la bandera anticorrupción que había enarbolado Simeón II, y que había resultado clave en su victoria electoral, generaba también una profunda decepción en el electorado como consecuencia de hechos como que algunos de sus ministros aparecieran implicados en actividades sospechosas. En definitiva, estos resultados electorales marcaban el fin de la esperanza depositada en la persona que llegaría a ser primer ministro de un país cuyo idioma no era capaz de hablar con fluidez.

El Partido Socialista, liderando la Coalición por Bulgaria, volvía al Gobierno del país después de dos mandatos en la oposición. Aparecían algunos protagonistas novedosos de la vida política búlgara, como los Demócratas por una Bulgaria Fuerte, una opción de muy definida sensibilidad anticomunista que lideraba el que fuera primer ministro Ivan Kostov. Sin embargo, la más destacada de entre las nuevas opciones era Ataka, un movimiento de extrema derecha que se convirtió en un importante motivo de preocupación. En cierto modo, el fenómeno Ataka no era realmente nuevo, en tanto que representaba otra forma de partidos o coaliciones anti. Como explican los profesores Tat- 
Tabla 15. Elecciones legislativas 2005. Resultados electorales

Votantes censados 6.720 .941

Votantes 3.747.793

Participación 55,08\%

\begin{tabular}{lccc}
\hline Partido o coalición & Votos & \% votos & Escaños \\
\hline Coalición por Bulgaria & 1.129 .196 & 33,98 & 82 \\
Movimiento Nacional Simeón II & 725.314 & 21,83 & 53 \\
Movimiento por los Derechos y las Libertades & 467.400 & 12,07 & 34 \\
Coalición Ataka & 296.848 & 8,93 & 21 \\
Fuerzas Democráticas Unidas & 280.323 & 8,44 & 20 \\
Demócratas por una Bulgaria Fuerte & 234.788 & 7,07 & 17 \\
Unión Popular Búlgara & 189.268 & 5,70 & 13 \\
Otros & 325.040 & 1,98 & 0 \\
\hline
\end{tabular}

Fuente: Central Electoral Commission of the Republic of Bulgaria (http://www.2005izbori.org/results/index. html).

yana Dronzina e Ivanka Mavrodieva, «los partidos "anti” son frecuentemente descritos como expresión del voto de protesta en contra del sistema existente o de las élites gobernantes, o como resultado de la crisis de la representación, que inclina a los votantes a no sentirse satisfactoriamente representados por ninguno de los actuales sujetos políticos [...]. Los partidos "anti" se nutren de los problemas reales no resueltos» (Dronzina y Mavrodieva, 2009: 36).

Los resultados de las elecciones presidenciales de 2006 quedaron definidos en base a dos características que podemos considerar innovadoras - una de ellas relacionada con Ataka. De un lado, los resultados rompían la tendencia dominante en Bulgaria desde las primeras elecciones democráticas de 1990, según la cual cada elección conllevaba un cambio en el signo político de la opción ganadora. En 2006, el presidente Georgi Parvanov fue elegido para un segundo mandato consecutivo, lo cual mostró un grado de conexión con el electorado que iba más allá del de su propio partido. Pero, de una manera también significativa, por primera vez quedaba rota la bipolaridad del panorama búlgaro de partidos políticos. El segundo candidato con más votos en la primera vuelta fue Siderov, el representante de Ataka. El ticket electoral Beronov/ Nikolova, que consiguió reunir el apoyo de los partidos más representativos de la derecha (excluida la más extremista), obtuvo sólo el 9,75 de los votos, y quedó excluido de la segunda vuelta.

De esta manera, encontramos una buena razón para entender por qué Ataka sembró tanta inquietud y generó tantos análisis. Antes de la celebración de la segunda vuelta, había una profunda preocupación acerca del nivel que pudiera alcanzar el apoyo popular a esta opción. Teniendo en cuenta la historia electoral del país - con cambios en el gobierno tan frecuentes- y el malestar en la población, se temía incluso que Ataka pudiera obtener la mayoría recogiendo el voto desesperado que surgía de la insatisfacción de las gentes de Bulgaria. Al final, Ataka obtuvo solo un 24,05 por ciento de los sufragios en la 
Tabla 16. Elecciones presidenciales 2006. Resultados electorales. Primera vuelta

\begin{tabular}{lrr}
\hline Votantes inscritos 7.075 .041 & & \\
Votos válidos 2.856 .734 & & \\
Participación 40,38\% & \multicolumn{1}{c}{ Votos } & \% votos \\
\hline Candidatos & 271.078 & 9,75 \\
\hline Beronov/Nikolova & 13.854 & 0,50 \\
Petrov/Topalova & 1.780 .119 & 64,05 \\
Parvanov/Marin & 19.857 & 0,71 \\
Velev/Mutafchiev & 21.812 & 0,79 \\
Beron/Angelova & 597.175 & 21,49 \\
Siderov/Shopov & 75.478 & 2,71 \\
Markov/Tsoneva & 2.779 .373 & 100,00 \\
Total & &
\end{tabular}

Fuente: Central Electoral Commission of the Republic of Bulgaria (http://www.izbori2006.org/results_1/ index.html).

Tabla 17. Elecciones presidenciales 2006. Segunda vuelta

Votantes inscritos 7.075.041

Votos válidos 2.757.441

Participación 38,97\%

\begin{tabular}{lrr}
\hline \multicolumn{1}{c}{ Candidatos } & \multicolumn{1}{c}{ Votos } & \% votos \\
\hline Parvanov/Marin & 2.050 .488 & 75,95 \\
Siderov/Shopov & 649.387 & 24,05 \\
TOTAL & 2.699 .875 & 100,00 \\
\hline
\end{tabular}

Fuente: Central Electoral Commission of the Republic of Bulgaria (http://www.izbori2006.org/results_2/ index.html).

segunda vuelta, y el socialista Georgi Parvanov consiguió una amplia victoria. Muy probablemente, el voto táctico en contra de Ataka desempeñó un papel significativo.

Por el contrario, los resultados de las elecciones generales de 2009 no fueron inesperados en absoluto. Desde hacía algún tiempo, la figura política de Boyko Borissov estaba logrando recoger las esperanzas de una buena parte de la población búlgara. El que otrora fuera deportista en la modalidad de lucha, se había establecido profesionalmente en el campo de la seguridad privada. De hecho, una de sus empresas tuvo la seguridad de Simeón II bajo su responsabilidad. Borissov concurrió a las elecciones a la alcaldía de Sofía y, tras obtener la victoria, la alcaldía le proporcionó la proyección necesaria para optar al puesto de primer ministro. No es posible encontrar en Boyko Borissov nada distinto de una persona cuyo rol se esperaba que fuese, más allá de ideologías, útil para solventar de manera expeditiva los problemas del país. En cierto modo, su figura tiene alguna similitud con la de Vladimir Putin. 
Tabla 18. Elecciones legislativas 2009. Resultados electorales (parte proporcional)

Votantes censados 7.129 .965

Votantes 4.345 .450

Participación 60,9\%

\begin{tabular}{lccc}
\hline Partido o coalición & Votos & \% votos & Escaños \\
\hline Ciudadanos para el Desarrollo Europeo de Bulgaria & 1.678 .641 & 39,7 & 116 \\
Coalición por Bulgaria & 784.147 & 17,7 & 40 \\
Movimiento por los Derechos y las Libertades & 610.521 & 14,4 & 38 \\
Coalición Ataka & 395.733 & 9,4 & 21 \\
Coalición Azul & 285.662 & 6,8 & 15 \\
Orden, Legalidad, Justicia & 174.582 & 4,1 & 10 \\
Líder & 137.795 & 3,3 & 0 \\
Movimiento Nacional por la Estabilidad y el Progreso & 127.470 & 3,0 & 0 \\
Otros & 67.644 & 1,6 & 0 \\
\hline
\end{tabular}

Fuente: Central Electoral Commission of the Republic of Bulgaria (http://rezultati.cik2009.bg/results/proportional/rik_00.html).

En las elecciones parlamentarias de 2009, entró en vigor un sistema electoral ligeramente modificado. Del total de 240 parlamentarios, 209 eran elegidos a través de un sistema de lista cerrada con voto proporcional, y 31 a través de un voto mayoritario en circunscripciones uninominales. Se establecen 31 distritos plurinominales en la elección por listas cerradas, y se mantenía la vigencia del mínimo del 4 por ciento de votos para acceder a la distribución de escaños. Cada circunscripción plurinominal es, a su vez, un distrito uninominal en la elección entre candidatos individuales. De este modo, cada votante dispone de dos votos.

Ciudadanos para el Desarrollo Europeo de Bulgaria (GERB, en sus iniciales búlgaras) consiguió más del doble de votos que la coalición entonces gobernante. En las circunscripciones uninominales, GERB obtuvo 26 escaños del total de 31, mientras que el Movimiento por los Derechos y las Libertades consiguió 5 de ellos. De esta manera, el panorama político de Bulgaria cambiaba, una vez más, de manera significativa. Ya no era posible encontrar la tradicional bipolaridad entre el campo socialista, de un lado, y la sensibilidad anticomunista, de otro. Nuevamente, los socialistas mostraron disponer de un suelo electoral —o un mínimo de votos en condiciones adversas — realmente sólido, y fue la segunda opción en número de votos, con ello mantuvo vivo el polo socialista. Sin embargo, la diferencia con Ciudadanos para el Desarrollo Europeo de Bulgaria — la opción de Borissov_ era elevada. Esta opción parecía heredar las características sociales de la base electoral que había definido anteriormente a la UFD y al Movimiento Nacional Simeón II. En la opinión pública, las expectativas de victoria de GERB eran elevadas, por su potencialidad para recoger lo que el profesor de Ciencia Política Antony Todorov denominaba «approval vote», según el cual el elector piensa que "es mejor dar una oportunidad al 
Tabla 19. Elecciones legislativas 2009. Distribución de escaños

\begin{tabular}{lccr}
\hline Partido o coalición & $\begin{array}{c}\text { Escaños } \\
\text { parte } \\
\text { proporcional }\end{array}$ & $\begin{array}{c}\text { Escaños } \\
\text { parte } \\
\text { mayoritaria }\end{array}$ & Total \\
\hline Ciudadanos para el Desarrollo Europeo de Bulgaria & 90 & 26 & 116 \\
Coalición por Bulgaria & 40 & - & 40 \\
Movimiento por los Derechos y Libertades & 33 & 5 & 38 \\
Coalición Ataka & 22 & - & 21 \\
Coalición Azul & 15 & - & 15 \\
Orden, Legalidad y Justicia & 10 & - & 10 \\
Líder & - & - & 0 \\
Movimiento Nacional para la Estabilidad y el Progreso & - & - & 0 \\
Otros & - & - & 0 \\
\hline
\end{tabular}

Fuente: Central Electoral Commission of the Republic of Bulgaria (http://rezultati.cik2009.bg/results/mandates/rik_00.html).

ganador emergente» ${ }^{9}$. Ataka mantuvo parte de su influencia, con 21 escaños en la Asamblea Nacional, pero dejó de ser la segunda fuerza política del país. El Movimiento por los Derechos y las Libertades consiguió un resultado superior al doble del obtenido en las primeras elecciones tras la caída del régimen comunista. En cierto modo, ello significaba que había llegado a ser algo más que un partido que representaba sólo a un grupo étnico concreto. Su actitud de responsabilidad, apoyando como partido bisagra la estabilidad del país, favoreció muy probablemente su imagen ante una buena parte del cuerpo electoral.

\section{Análisis estadísticos basados en datos de nivel de distrito}

Aunque hemos usado los resultados electorales al nivel nacional como un indicador fundamental en nuestros análisis, nos centraremos ahora en los resultados a nivel de distrito, con el propósito, de un lado, de observar en qué medida aparecen influenciados por diversos factores sociológicos, $\mathrm{y}$, de otro lado, desde la perspectiva de analizar la existencia de una continuidad en las bases electorales de las opciones que emergieron, de manera exitosa, después de 2011. A tales fines, hemos tomado los siguientes indicadores al nivel de distrito: tasa de pobreza, densidad de población por kilómetro cuadrado, proporción de población urbana, tasa de desempleo y media salarial anual. Hemos usado los datos disponibles de 1999 y 2007 de cara a correlacionar los resultados electorales con el contexto social apropiado. Este detalle no es especialmente relevante en lo que se refiere al análisis de correlación de Pearson, porque los cambios en los datos socioeconómicos entre 1999 y 2007 tienen lugar en un sentido similar en todos los distritos. Sin embargo, hemos considerado que era más apropiado

9. Novinite.com (http://www.novinite.com/view_news.php?id=105500, consulta: 7 julio 2009). 
Tabla 20. Distritos búlgaros

\begin{tabular}{llll}
\hline Vidin & Razgrad & Burgas & Sofia \\
Vratsa & Russe & Sliven & Sofia cap*. \\
Lovech & Silistra & Stara Zagora & Kardzhali \\
Montana & Varna & Yambol & Pazardzhik \\
Pleven & Dobrich & Blagoevgrad & Plovdiv $^{\star *}$ \\
Veliko Tarnovo & Targovishte & Kyustendil & Smolyan \\
Gabrovo & Shumen & Pernik & Haskovo \\
\hline
\end{tabular}

* Circunscripciones Sofia 23, Sofia 24 y Sofia 25.

** Circunscripciones Plovdiv 16 y Plovdiv 17.

Fuente: Central Electoral Commission of the Republic of Bulgaria.

Tabla 21. Correlaciones variables. Indicadores socioeconómicos. Por distritos

\begin{tabular}{lcccc}
\hline & Tasa pobreza & Desempleo 1999 & Salarios 1999 & Densidad 1999 \\
\hline Desempleo 1999 & 0,696 & & & \\
Salarios 1999 & $-0,729$ & $-0,542$ & & \\
Densidad 1999 & $-0,668$ & 0,523 & 0,431 & \\
Urbana 1999 & $-0,781$ & $-0,656$ & 0,591 & 0,576 \\
\hline & Tasa pobreza & Desempleo 2007 & Salarios 2007 & Densidad 2007 \\
\hline Desempleo 2007 & 0,687 & & & \\
Salarios 2007 & $-0,811$ & $-0,567$ & & \\
Densidad 2007 & $-0,674$ & $-0,476$ & 0,788 & \\
Urbana 2007 & $-0,764$ & $-0,777$ & 0,593 & 0,576 \\
\hline
\end{tabular}

Fuente: cálculos propios a partir de datos del National Statistical Institute.

correlacionar resultados electorales con datos socioeconómicos del correspondiente marco temporal. Hemos correlacionado los resultados electorales de 2001 con los datos socioeconómicos por distritos de 2007. Ciertamente, en términos puramente cronológicos, 2001 se encuentra más cercano a 1999. Sin embargo, en 2001 comienza un nuevo periodo económico en Bulgaria, definido como de recuperación económica gradual (AAVV, 2003: 9), y hemos estimado más apropiado vincular estadísticamente los resultados electorales de 2001 con los indicadores socioeconómicos de 2007. En la tabla 20, recogemos los distritos de Bulgaria.

En la tabla 21, ofrecemos los índices de correlación de Pearson entre los mencionados indicadores, para hacer visible su interconexión en la vida social de Bulgaria.

El gráfico 1 muestra, en forma de diagramas de caja (o boxplots), los datos de los indicadores socioeconómicos por distritos que hemos utilizado, mostrando de una manera visual las características más básicas de esta información. Quisiera 
Gráfico 1. Indicadores socioeconómicos por distritos
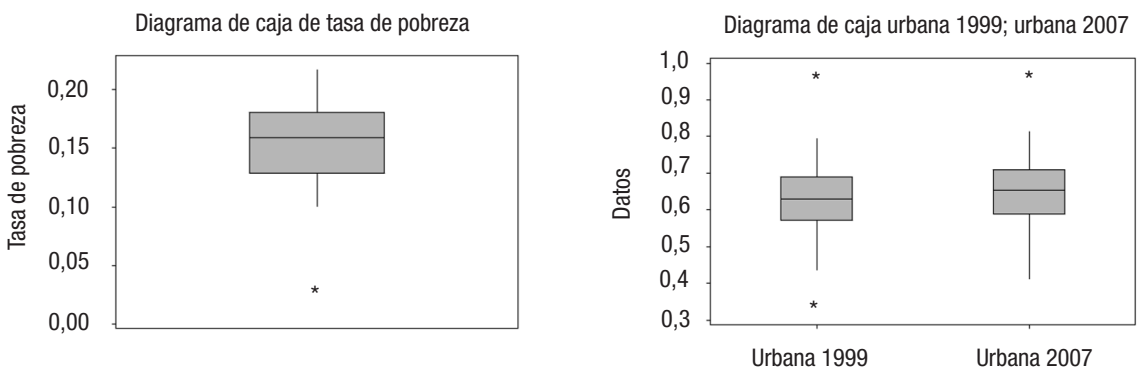

Diagrama de caja de densidad 1999; densidad 2007

Diagrama de caja de salarios 1999; salarios 2007
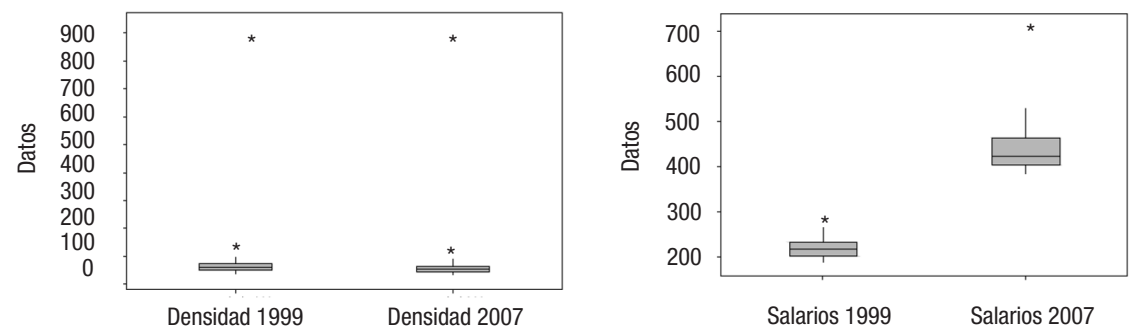

Diagrama de caja de desempleo 1999; desempleo 2007

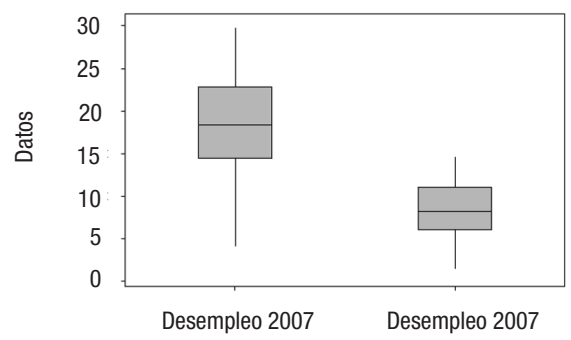

Fuente: cálculos propios a partir de datos del National Statistical Institute.

señalar la existencia de un outlier recurrente, se trata del distrito de Sofía ciudad, con características socioeconómicas especialmente marcadas en contraste con el resto. Este hecho es especialmente visible en lo que se refiere a densidad de población, pero también aparece en el resto de indicadores. Es importante tener en cuenta estas características especiales de la capital del país, por su elevado potencial analítico. Sin este outlier, los rangos intercuartílicos, expresados numéricamente, podrían haber sido unos indicadores útiles para describir nuestros datos, pero, debido a esta peculiaridad estadística, los diagramas de caja resultan ser unas opciones más apropiadas para visualizar la información. 


\subsection{Análisis de correlación: la influencia socioeconómica}

Así pues, presentamos esta información de carácter socioeconómico en correlación con los resultados electorales de las elecciones parlamentarias, aportando de esta manera una aproximación a la influencia de la realidad social en el comportamiento electoral. Un punto de interés principal ha sido el tratar de observar en qué medida las opciones electorales que representaban una apertura a los nuevos tiempos tenían sus bases electorales en grupos sociodemográficos de los que normalmente se esperaría que mostrasen una menor reticencia ante los cambios. En este sentido, hemos tratado de observar cómo relevantes opciones del polo no socialista, como las encabezadas por el anterior rey Simeón II y el actual primer ministro Boyko Borissov, aparecen sociológicamente arraigadas en los entornos propicios a los partidos y a las coaliciones que más nítidamente definían sus señas de identidad en la oposición al régimen comunista — con la amplia diversidad de connotaciones que ello conllevaba en la sociedad búlgara.

En términos sociodemográficos, es posible encontrar algunas líneas de división (o cleavages) en lo que se refiere al apoyo a las principales opciones electorales de Bulgaria. Es algo que puede ser apreciado mediante el uso de correlaciones variables (tabla 22). El voto a la UFD muestra unas claras correlaciones negativas con tasa de pobreza y desempleo (cuanto más elevados son estos indicadores, menor es el apoyo electoral a la UFD, y viceversa), y correlaciones positivas, siguiendo una misma lógica, con salarios, densidad y proporción de población urbana. Observamos también que, a nivel de distrito, el PSB aparece representado en estos dígitos con rasgos menos definidos, debido a su distribución geográfica más homogénea, y también al hecho de que comparte entornos con el Movimiento por los Derechos y las Libertades - la opción representativa de la minoría de etnia turca, de presencia igualmente amplia en las zonas rurales. Con todo, el perfil sociodemográfico básico del PSB ha sido también muy característico ${ }^{10}$. Es algo que se ha reflejado ampliamente en encuestas de opinión de todo tipo, aunque esta realidad queda muy bien sintetizada en el siguiente documento del Centro para el Estudio de la Democracia:

La estructura educativa de los votantes de las dos opciones principales es de sumo interés, porque se aprecia una clara correlación entre nivel educativo y actitudes políticas. En general, las preferencias a favor del PSB disminuyen a medida que aumenta el nivel educativo. $\mathrm{Y}$ viceversa, el incremento en el nivel educativo correlaciona con el incremento en las preferencias a favor de la UFD. El 50 por ciento de los entrevistados con titulación de Bachiller preferían que ganase la UFD, frente al 39 por ciento que optaban por el PSB. [...] mientras que el 52 por ciento de los entrevistados con educación básica declaraban su apoyo al PSB.

10. En relación con el género como factor explicativo, su papel no es tan significativo como en otros países europeos. La profesora Petya Pachkova ha puesto de relieve que en Bulgaria no pueden apreciarse diferencias relevantes en el comportamiento electoral femenino (Pachkova, 1996: 25-26). Los aspectos de este libro específicamente relacionados con el comportamiento electoral pueden verse en el capítulo «Electoral behaviour during political transition» (Pachkova, 1997). 
Tabla 22. Correlaciones variables. Voto a coalición o partido/indicadores sociales. Por distritos

\begin{tabular}{|c|c|c|c|c|c|c|c|c|}
\hline & \multicolumn{2}{|c|}{1990} & \multicolumn{2}{|c|}{1991} & \multicolumn{2}{|c|}{1994} & \multicolumn{2}{|c|}{1997} \\
\hline & PSB & UFD & PSB & UFD & PSB & UFD & PSB & UFD \\
\hline Tasa pobreza & 0,074 & $-0,694$ & 0,053 & $-0,689$ & 0,108 & $-0,703$ & 0,011 & $-0,499$ \\
\hline Desempleo 1999 & 0,445 & $-0,644$ & 0,354 & $-0,635$ & 0,457 & $-0,667$ & 0,275 & $-0,466$ \\
\hline Salarios 1999 & $-0,088$ & 0,488 & $-0,062$ & 0,442 & $-0,137$ & 0,492 & $-0,004$ & 0,401 \\
\hline Densidad 1999 & $-0,215$ & 0,454 & $-0,207$ & 0,397 & $-0,324$ & 0,542 & $-0,197$ & 0,312 \\
\hline \multirow[t]{3}{*}{ Urbana 1999} & 0,168 & 0,803 & 0,113 & 0,765 & $-0,041$ & 0,777 & 0,032 & 0,652 \\
\hline & \multicolumn{2}{|c|}{2001} & \multicolumn{2}{|c|}{2007} & \multicolumn{2}{|c|}{2009} & & \\
\hline & PSB & MNSII & CB & MMSII & $\mathrm{CB}$ & GERB & & \\
\hline Tasa pobreza & $-0,192$ & $-0,429$ & 0,038 & $-0,053$ & $-0,171$ & $-0,636$ & & \\
\hline Desempleo 2007 & 0,158 & $-0,448$ & 0,426 & 0,106 & 0,114 & $-0,662$ & & \\
\hline Salarios 2007 & 0,065 & 0,200 & $-0,100$ & 0,000 & 0,031 & 0,431 & & \\
\hline Densidad 2007 & $-0,051$ & 0,054 & $-0,184$ & 0,023 & $-0,061$ & 0,301 & & \\
\hline Urbana 2007 & 0,142 & 0,674 & $-0,166$ & 0,180 & 0,241 & 0,835 & & \\
\hline
\end{tabular}

Fuente: cálculos propios a partir de datos del National Statistical Institute, del profesor Dimitar Dimitrov y de la Central Electoral Commission of the Republic of Bulgaria.

Hay una relativamente clara interdependencia entre afiliación política y edad. Los mayores niveles de edad correlacionan con cifras igualmente elevadas de electores que apoyan al PSB. Esto queda reflejado también en el nivel educativo: los electores con un nivel sólo básico o inferior son, en su mayor parte, gente de edad avanzada. En los partidarios de la UFD encontramos la correlación opuesta. Entre los encuestados, los votantes menores de 45 años constituyen el 72 por ciento del total de los partidarios de esta opción. Por debajo de este mismo nivel de edad, se ubica el 48 por ciento de los que apoyan al PSB. (Center for the Study of Democracy, 1990)

Después de la irrupción del Movimiento Nacional Simeón II en la escena política búlgara, esta opción parece heredar los rasgos sociodemográficos de la UFD, con mayor apoyo en los entornos más caracterizadamente urbanos y un menor respaldo en donde el desempleo y las tasas de pobreza son más elevados. Sin embargo, con la decepción que aparece después de esos momentos iniciales, los claros perfiles que observábamos en las correlaciones se difuminan, como si sugirieran que incluso las expectativas que había generado en sus entornos más propicios tendían a desaparecer.

\subsection{Análisis de regresión: continuidad MNSII-GERB}

Lo que ha atraído la atención de los observadores hacia el caso búlgaro ha sido el hecho de que dos opciones fraguadas en torno a unas fuertes figuras personales hubieran emergido, de una manera electoralmente exitosa, como formas de intentar superar la inagotable crisis económica. Resulta de interés comprobar en qué medida una de estas opciones se fundamenta en la otra - $\mathrm{O}$, en otras 
Gráfico 2. Gráfico de probabilidad normal de los residuos

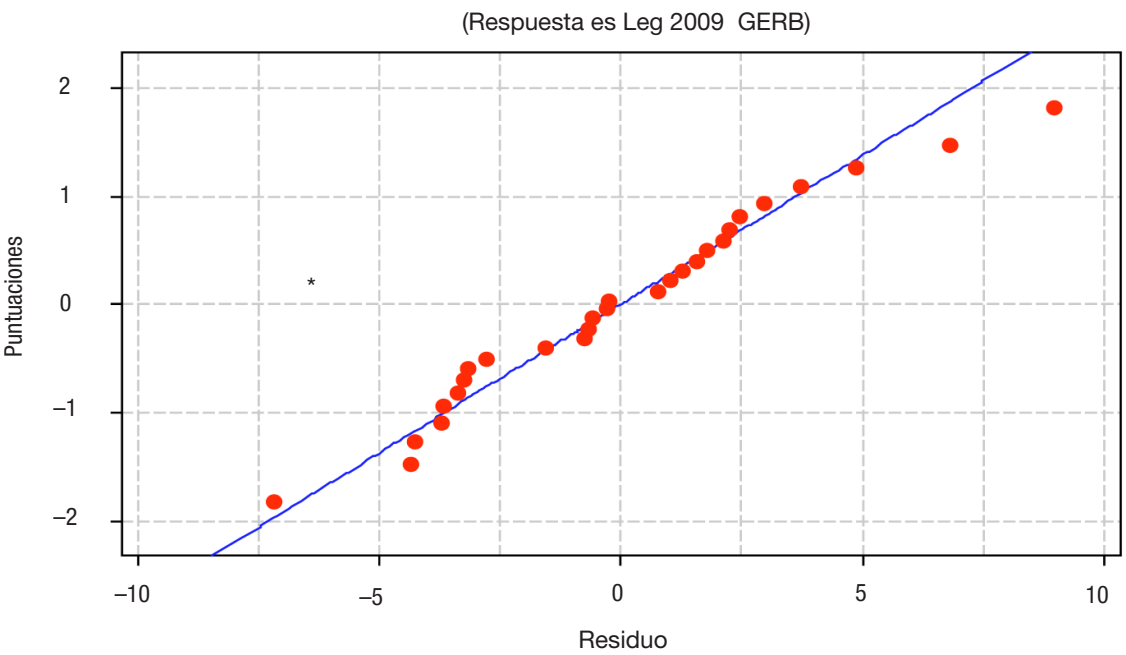

Fuente: cálculos propios a partir de datos de la Central Electoral Commission of the Republic of Bulgaria.

palabras, hasta qué punto el Movimiento Nacional Simeón II se encuentra en la base del origen de GERB. Hemos llevado a cabo un análisis de regresión lineal para comprobar la hipótesis de que no hay relación entre el voto al MNSII por circunscripciones, como variable explicativa, y el voto a GERB en ese mismo nivel, como variable respuesta - es decir, $H O: \beta=0$. De cara a verificar los requisitos de normalidad del modelo, de un lado, y que los residuos provienen de distribuciones con media cero y varianza constante, de otro, hemos generado un gráfico de probabilidad normal y un gráfico de residuos.

Dado que los puntos en el gráfico de probabilidad normal de los residuos se encuentran próximos a la línea recta, podemos asumir que un modelo normal es apropiado. Y no es posible encontrar ninguna pauta definida en la distribución de los puntos del gráfico de residuos. Aparecen irregularmente distribuidos en torno a cero, lo que hace que podamos considerar como perfectamente plausible el segundo requisito enunciado. De esta manera, como los dos requisitos para utilizar un modelo de regresión variable quedan satisfechos, podemos considerarlo como apropiado para estos datos.

Los cálculos dan el resultado expresado en la tabla 23.

La última columna de la tabla $23, P$, ofrece en las correspondientes filas los valores $p$ para $H 0: \alpha=0$ (fila superior) y $H O: \beta=0$ (fila inferior). Por lo tanto, el valor $p$ que probaría la hipótesis de que el 2001 NMSII voto no tiene efecto sobre 2009 GERB voto es 0,000. De esta manera, como 0,000 es $<0,01$, encontramos una fuerte evidencia en contra de la hipótesis de que el nivel de apoyo electoral a MNSII en 2001 no influye en el que obtiene GERB en 2009. 
Gráfico 3. Residuos versus valores ajustados

(Respuesta is Leg 2009 GERB)

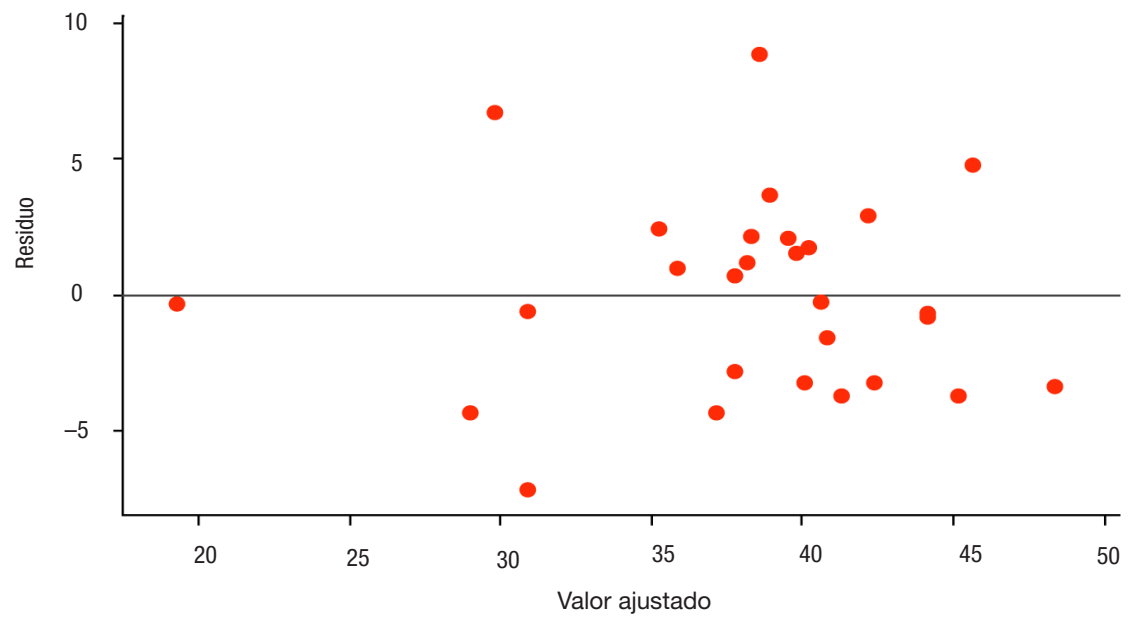

Fuente: cálculos propios a partir de datos de la Central Electoral Commission of the Republic of Bulgaria.

Tabla 23. Análisis de regresión: Leg 2009 GERB versus leg 2001 Simeón

La ecuación de regresión es

Leg 2009 GERB = 9,07 + 0,691 leg 2001 Simeón

\begin{tabular}{lllll}
\multicolumn{1}{c}{ Predictor } & Coef SE & Coef & T & P \\
\hline Constant & 9,066 & 3,526 & 2,57 & 0,016 \\
Leg 2001 Simeón & 0,69083 & 0,08178 & 8,45 & 0,000 \\
\hline
\end{tabular}

Fuente: cálculos propios a partir de datos de la Central Electoral Commission of the Republic of Bulgaria.

\section{Conclusión: contrastando hipótesis a lo largo de veinte años de vida democrática}

Mediante nuestros análisis, hemos tenido ocasión de observar cómo los resultados electorales pueden ser indicadores realmente útiles de los sentimientos y las necesidades de los ciudadanos de Bulgaria. Brevemente, podemos señalar dos flujos electorales básicos en función de la actitud del elector ante las urnas. De un lado, el núcleo duro de cada uno de los lados del básicamente bipolar panorama de partidos búlgaro - $\mathrm{o}$, mas bien, tripolar, si pensamos en el habitualmente disciplinado electorado perteneciente a la etnia turca. Sobre esta base, encontramos a un electorado muy consistentemente imantado a cada uno de los polos. En el caso del PSB, ello se traduce en los comparativamente elevados suelos que alcanza en elecciones de contexto tan dramático como las de 1997 — cuando recoge un 22,07 por ciento de 
los votos-o en las de 2001 - con un 17,15 por ciento-, con situaciones políticas que no eran en absoluto favorables a los socialistas. Junto a este comportamiento de fidelidad, observamos, de un lado, un caudal de voto (o, algunas veces, de no-voto) de desesperanza orientado en un sentido triple: hacia nuevas y atípicas opciones como las representadas por Simeón SajoniaCoburgo, Boyko Borissov o, sin tanta fortuna electoral, Georges Ganchev; hacia la alternancia en el gobierno, o hacia la abstención. En otras palabras, la falta de estabilidad social en Bulgaria tiene su correlato en la falta de estabilidad política y electoral. Esta situación tiene su traducción correspondiente en un mapa electoral altamente volátil, en el que la alternancia - tanto en el gobierno como en el papel de opción victoriosa electoralmente- llega a ser una constante, con muy pocas excepciones.

Somos perfectamente conscientes de la elevada complejidad intrínseca a una sociedad en transición, con una diversidad de factores - papel y comportamiento de las élites, influencia de la cultura política...- que merecerían por sí mismos un análisis, al menos, tan profundo como el ofrecido en estas páginas. En cualquier caso, desde la perspectiva que hemos asumido para esta investigación, es posible concluir, sintéticamente, que hay un factor que late intensamente en la transición búlgara, que condiciona el comportamiento de los ciudadanos de este país ante las urnas. Este factor puede ser expresado en la idea de desesperanza, como proponíamos en nuestra tesis central inicial. Después de veinte años de elecciones democráticas, es aún difícil ver la luz al final del túnel.

Hablando en términos generales, este factor influye en los movimientos electorales de péndulo observados y en el surgimiento de opciones políticas atípicas, pero, más específicamente, condiciona como cada sector social comprende la vida política, ligando el voto popular a una elección racional en una manera que podemos considerar que confirma nuestra primera hipótesis. El pragmatismo que inspira la interminable búsqueda electoral de una solución a las dificultades de la vida cotidiana puede ser razonablemente interpretado sobre la base de una racionalidad en la elección del voto, a la manera de un cálculo sobre el coste y el beneficio.

Por lo que se refiere a la segunda hipótesis, además de observar algunos datos e informaciones procedentes de centros de investigación de la opinión pública que vinculan voto popular con pertenencia a grupos sociales, hemos comprobado, mediante índices de correlación de Pearson, en qué medida el voto por distritos correlaciona con significativos indicadores sociodemográficos a ese mismo nivel. Ello es cierto en relación con el voto en elecciones parlamentarias hacia opciones que representan la oposición al polo socialista / ex comunista. La correlación pierde peso, de un lado, cuando se trata del voto socialista en elecciones legislativas, porque el PSB comparte entorno geográfico con el partido o la coalición representativo de la minoría de etnia turca, y, de otro lado, porque el voto al PSB aparece más homogéneamente distribuido en los distintos tipos de entornos, mientras que el voto a la UFD - $\mathrm{o}$ a sus herederos- muestra unos rasgos mucho más marcados geográficamente. En términos generales, puede afirmarse que la realidad socioeconómica búlgara 
subyace en el conjunto de los resultados electorales, y cómo los diferentes grupos afrontan los problemas de la transición condiciona la forma en que desarrollan sus actitudes políticas y su comportamiento electoral.

Por lo que respecta a la tercera hipótesis, hemos mostrado, a través de un análisis de regresión cómo opciones exitosas, como las lideradas por el antiguo rey Simeón II o el actual primer ministro Borissov, muestran una continuidad estadística - ambas comparten bases sociales con otras opciones que simbolizan la cultura de rechazo al régimen comunista y, en consecuencia, de aceptación de los cambios y los nuevos tiempos.

Podemos, de esta manera, concluir que el contexto homogéneo que aportan veinte años de vida en democracia y con economía de mercado nos ofrece un fértil laboratorio en el que analizar la validez de los factores explicativos del comportamiento electoral, y, junto a ello, nos aporta una perspectiva contemporánea de la realidad del voto en un contexto de continuada conflictividad y malestar social. Confiemos en que estos análisis no sean nunca aplicables a la sociedad española, ni a ninguna otra, como consecuencia de los devenires de los mercados financieros...

\section{Referencias bibliográficas}

Center for the Study of Democracy (1990). The Political Change In Bulgaria Pre-Electoral Attitudes, June 7, 1990 [en línea]. <http://www.csd.bg/artShow. php?id=13736> [Consulta: 15 mayo 2010].

- (1992). Public Opinion and Bulgarian Business Elite on Privatization, 1992 [en línea]. <http://www.csd.bg/artShow.php?id=10436> [Consulta: 15 mayo 2010].

Dimitrova, Boriana (1997). «Nesastoiolata se sotzialdemokratzia». En: VVAA Balgarskite Izbori 1990-1996. Rezultati, analizi, tendentsii. Sofia: Izdatelsbo Demokratichni Traditsii-Demetra.

Downs, Anthony (1957). «An Economic Theory of Political Action in a Democracy». The Journal of Political Economy, 65 (2), 135-150.

Dronzina, Tatyana y Mavrodieva, Ivanka Mavrodieva (2009). «El surgimiento de Ataka: ¿vuelta del nacionalismo o llegada del antiglobalismo a la escena búlgara?». En: Flores, Carlos (ed.). España y la Europa Oriental: tan lejos, tan cerca. Valencia: Universidad de Valencia.

European Commission (2002). Candidate Countries Eurobarometer 2001 (anexos), marzo. Bruselas.

Genov, Nikolai (1995). Bulgaria. Human Development Report 1995. Sofia: Regional and Global Development.

Giatzidis, Emil (2002). An introduction to postcommunist Bulgaria: political, economic and social transformation. Manchester: Manchester University Press.

Harrop, Martin y Miller, William (1987). Elections and Voters: A comparative introduction. Nueva York: New Amsterdam Books.

KirchHeimer, Otto (1996). «The transformation of West European Party Systems». En: Lapalombara, Joseph y Weiner, Myron (eds.). Political Parties and Political Development. Princeton: Princeton University Press.

Lipset, Seymour (1981). Political Man: The Social Bases of Politics. Maryland: The John Hopkins University Press. 
Mcintyre, Robert (1988). Bulgaria: economy, politics, society. Londres: Pinter Press. PachKova, Petya (1996). Elitat v Svetlitana na Izborite. Sofia: M-8-M.

- (1997). «Electoral behaviour during political transition». En: Karasimeonov, Georgi (ed.). The 1990 Election to the Bulgarian Grand National Assembly and the 1991 Election to the Bulgarian National Assembly. Berlín: Sigma.

Rojo, Luis Ángel (1990). «La URSS, sin plan y sin mercado». Claves de la Razón Práctica, 1, abril, 26-32.

Veiga, Francisco (1998). «Transiciones a la balcánica: ensayo de periodización y factores recurrentes». Papeles de la FIM, 9 (2. época), 63-80.

VVAA (2003). Bulgaria: the challenges of poverty. Sofia: National Statistical Institute. YANAKIEV, Yantsislav (2008). «Societal integration of the Turkish minority in Bulgaria». En: Genov, Nikolai (ed.). Interethnic Integration in five European societies. Hamburgo: Krämer. 


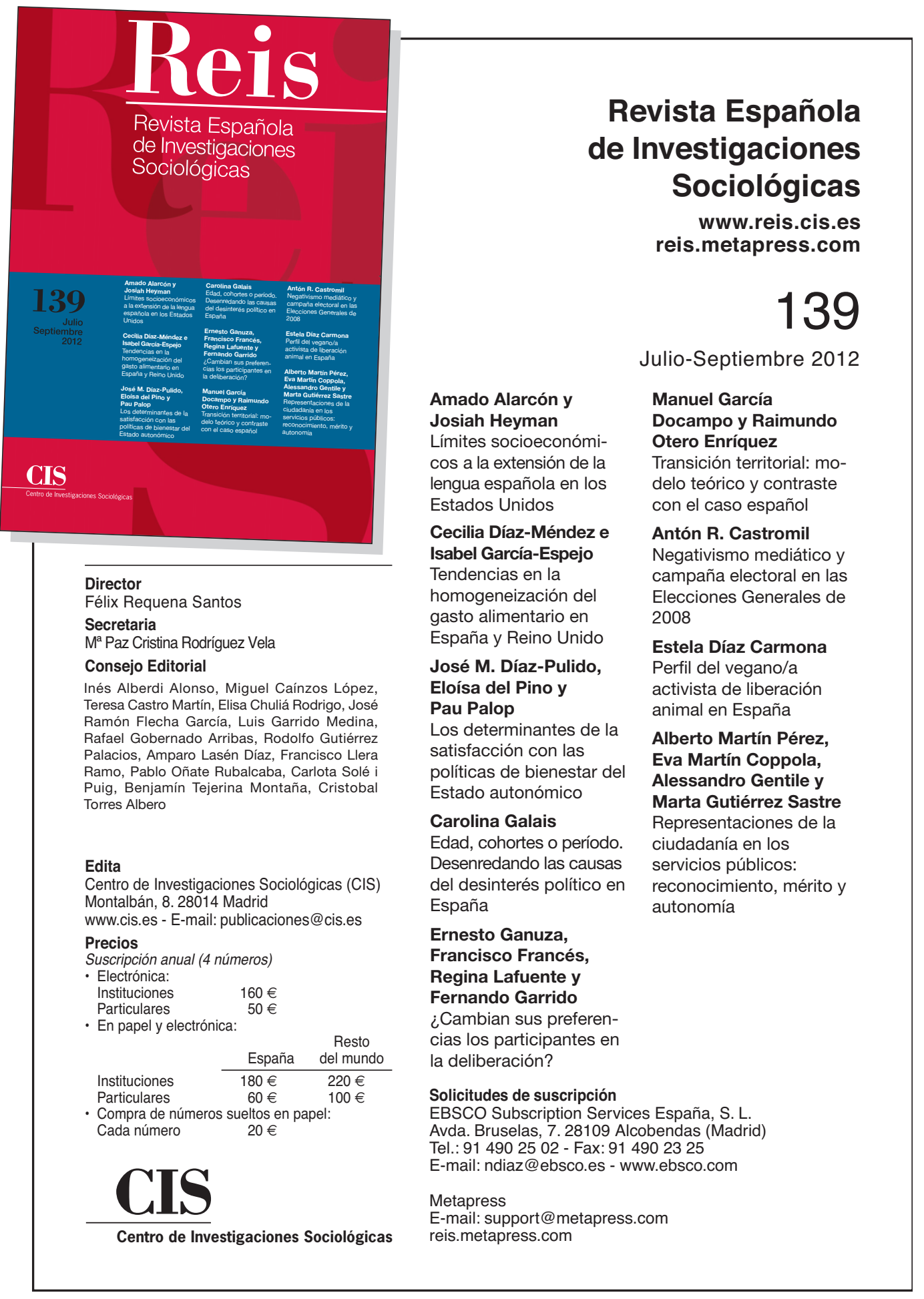

